Article

\title{
Generalized Almost Periodicity in Lebesgue Spaces with Variable Exponents, Part II
}

\author{
Marko Kostić ${ }^{1}$ (D) and Wei-Shih Du ${ }^{2, *}$ (ID \\ 1 Faculty of Technical Sciences, University of Novi Sad, Trg D. Obradovića 6, 21125 Novi Sad, Serbia; \\ marco.s@verat.net \\ 2 Department of Mathematics, National Kaohsiung Normal University, Kaohsiung 82444, Taiwan \\ * Correspondence: wsdu@mail.nknu.edu.tw
}

Received: 4 June 2020; Accepted: 25 June 2020; Published: 30 June 2020

\begin{abstract}
In this paper, we introduce and analyze several different notions of almost periodic type functions and uniformly recurrent type functions in Lebesgue spaces with variable exponent $L^{p(x)}$. We primarily consider the Stepanov and Weyl classes of generalized almost periodic type functions and generalized uniformly recurrent type functions. We also investigate the invariance of generalized almost periodicity and generalized uniform recurrence with variable exponents under the actions of convolution products, providing also some illustrative applications to the abstract fractional differential inclusions in Banach spaces.
\end{abstract}

Keywords: Weyl uniformly recurrent functions with variable exponents; quasi-asymptotically uniformly recurrent functions with variable exponents; quasi-asymptotically almost periodic functions with variable exponents; $S$-asymptotically $\omega$-periodic functions with variable exponents; Lebesgue spaces with variable exponents

MSC: primary 43A60; 35B15; 47D06

\section{Introduction and Preliminaries}

The notion of almost periodicity was first studied by Bohr around 1925 and later generalized by Stepanov, Weyl, and Besicovitch, amongst many others. There is an enormous amount of literature devoted to the study of various types of almost periodic functions and almost automorphic functions in Banach spaces. Fairly complete information concerning almost periodic functions, almost automorphic functions, and their applications can be obtained by consulting the research monographs by Besicovitch [1], by Diagana [2], by Fink [3], by Guérékata [4], by Kostić [5], by Levitan [6], and by Zaidman [7].

The theory of generalized almost periodicity and generalized uniform recurrence in Lebesgue spaces with variable exponents is still very unexplored. In their foundational papers [8,9], Diagana and Zitane introduced and analyzed the notion of Stepanov $p(x)$-pseudo almost periodicity and the notion of Stepanov $p(x)$-pseudo almost automorphicity. The spaces introduced in these papers are not translation invariant, in contrast to the spaces of Stepanov $p(x)$-almost periodic functions and Stepanov $p(x)$-almost automorphic functions considered by Diagana and Kostić in Reference [10,11]. The class of (asymptotically) Weyl almost periodic functions in Lebesgue spaces with variable exponents has been analyzed in Reference [12], while the classes of Stepanov uniformly recurrent functions, Doss uniformly recurrent functions, and Doss almost periodic functions in Lebesgue spaces with variable exponents were analyzed in the first part of this research study by Kostić and Du [13].

As mentioned in the abstract, the main aim of this paper was to analyze several different notions of almost periodic type functions and uniformly recurrent type functions in Lebesgue spaces with 
variable exponents. The paper is conceptualized as a certain continuation of our recent research studies [13,14], where we recently analyzed quasi-asymptotically almost periodic functions and their Stepanov generalizations (also see Reference [15]). The class of quasi-asymptotically uniformly recurrent functions has not been considered elsewhere; it is also worth noting that some classes of generalized Stepanov and Weyl $p(x)$-almost periodic type functions and $p(x)$-uniformly recurrent type functions have not been considered elsewhere even for the constant coefficients $p(x) \equiv p \in[1, \infty)$. We also examine the corresponding classes of functions depending on two parameters and clarify some composition principles in this context, providing a few illustrative applications to the abstract (semilinear) fractional differential inclusions in Banach spaces.

The organization and main ideas of this paper can be briefly described as follows. In Section 1.1, we collect the preliminary material about almost periodic functions and uniformly recurrent functions. Section 2 introduces the notion of several different types of generalized (equi-)Weyl almost periodicity in Lebesgue spaces with variable exponents. The spaces introduced in Definitions 3-5 may not be translation invariant, in general, which is not the case with the spaces introduced in Definitions 6-8. The main aim of Section 2 was to explain without proofs how the structural results and characterizations established for generalized (equi-)Weyl almost periodic functions in Reference [12] can be straightforwardly extended for the corresponding classes of generalized (equi-)Weyl uniformly recurrent functions. In Definition 9, we introduce the class of quasi-asymptotically uniformly recurrent functions. Proposition 1 shows that any asymptotically uniformly recurrent function is quasi-asymptotically uniformly recurrent; the converse statement is generally false, as a class of very simple counterexamples shows. In Proposition 2, we prove that the sum of a quasi-asymptotically uniformly recurrent function and a continuous function vanishing at infinity is again quasi-asymptotically uniformly recurrent. In Theorem 1 , we revisit Reference [14] (Theorem 2.5) once more and examine some extra conditions under which a quasi-asymptotically uniformly recurrent function is (asymptotically) uniformly recurrent. Section 3.1 introduces and investigates several different classes of Stepanov quasi-asymptotically uniformly recurrent type functions in Lebesgue spaces with variable exponents. The notion introduced in this subsection, in which we reconsider and slightly improve several known results from [14] in our new framework, is new even for the constant coefficients $p(x) \equiv p \in[1, \infty)$, and can be used to intermediate the concepts of the quasi-asymptotical almost periodicity (quasi-asymptotical uniform recurrence, S-asymptotical $\omega$-periodicity) and its Stepanov generalizations with constant exponents. In Proposition 4, we reconsider the assertion of Reference [10] (Proposition 4.5) for the Stepanov quasi-asymptotically uniformly recurrent functions (also see Corollary 1 and Proposition 5). It is worth noting that the concept of Stepanov $p$-quasi-asymptotical almost periodicity is important since it intermediates the concepts of the (quasi-)asymptotical almost periodicity and Weyl $p$-almost periodicity (see Reference [14], Proposition 2.12); as observed here, the same holds for the concept of Stepanov $p$-quasi-asymptotical uniform recurrence. The main objective in Proposition 6 is to state and prove a general result in this direction. In Section 3.2, we clarify the main composition principles for the class of quasi-asymptotically uniformly recurrent functions. Our main contributions are given in Section 3.3, where we examine the invariance of generalized quasi-asymptotical uniform recurrence with variable exponents under the actions of convolution products. Section 4 is reserved for applications of our abstract theoretical results to the abstract Volterra integro-differential inclusions in Banach spaces.

We use the standard notation throughout the paper. By $(X,\|\cdot\|)$, we denote a complex Banach space; unless stated otherwise, $I$ denotes the interval $[0, \infty)$ or the real line $\mathbb{R}$. Furthermore, by $L_{l o c}^{p}(I$ : $X), C(I: X), C_{b}(I: X)$, and $C_{0}(I: X)$, we denote the vector spaces consisting of all $p$-locally integrable functions $f: I \rightarrow X$, all continuous functions $f: I \rightarrow X$, all bounded continuous functions $f: I \rightarrow X$ and all continuous functions $f: I \rightarrow X$ satisfying that $\lim _{|t| \rightarrow+\infty}\|f(t)\|=0$, respectively $(1 \leq p<\infty)$. Then, $C_{0}(I: X)$ and $C_{b}(I: X)$ are Banach space equipped with the sup-norm, denoted henceforth by $\|\cdot\|_{\infty}$. If $f: \mathbb{R} \rightarrow X$, then we define $\check{f}: \mathbb{R} \rightarrow X$ by $\check{f}(x):=f(-x), x \in \mathbb{R}$. Furthermore, if $Y$ is also a complex Banach space, then, by $L(X, Y)$, we denote the space of all continuous linear 
mappings from $X$ into $Y ; L(X) \equiv L(X, X)$. For any $s \in \mathbb{R}$, we define $\lfloor s\rfloor:=\sup \{l \in \mathbb{Z}: s \geq l\}$ and $\lceil s\rceil:=\inf \{l \in \mathbb{Z}: s \leq l\}$.

Suppose that $f:[a, b] \rightarrow \mathbb{R}$ is a non-negative Lebesgue-integrable function, where $a, b \in \mathbb{R}, a<b$, and $\phi:[0, \infty) \rightarrow \mathbb{R}$ is a convex function. Let us recall that the Jensen integral inequality states that

$$
\phi\left(\frac{1}{b-a} \int_{a}^{b} f(x) d x\right) \leq \frac{1}{b-a} \int_{a}^{b} \phi(f(x)) d x
$$

Using this integral inequality, we can simply prove that, for every two sequences $\left(a_{k}\right)$ and $\left(x_{k}\right)$ of non-negative real numbers such that $\sum_{k=0}^{\infty} a_{k}=1$, we have

$$
\phi\left(\sum_{k=0}^{\infty} a_{k} x_{k}\right) \leq \sum_{k=0}^{\infty} a_{k} \phi\left(x_{k}\right)
$$

If $\phi:[0, \infty) \rightarrow \mathbb{R}$ is a concave function, then the above inequalities reverse.

\subsection{Almost Periodic and Uniformly Recurrent Type Functions}

Let $f: I \rightarrow X$ be a given continuous function. Given $\varepsilon>0$, we call $\tau>0$ an $\varepsilon$-period for $f(\cdot)$ if and only if $\|f(t+\tau)-f(t)\| \leq \varepsilon, t \in I$. The set consisting of all $\varepsilon$-periods for $f(\cdot)$ is denoted by $\vartheta(f, \varepsilon)$. The function $f$ is said to be almost periodic, if and only if for each $\varepsilon>0$ the set $\vartheta(f, \varepsilon)$ is relatively dense in $[0, \infty)$, which means that there exists $\ell>0$ such that any subinterval of $[0, \infty)$ of length $\ell$ intersects $\vartheta(f, \varepsilon)$. The collection of all almost periodic functions will be denoted by $A P(I: X)$. The function $f: I \rightarrow X$ is said to be asymptotically almost periodic if and only if there exist an almost periodic function $h: I \rightarrow X$ and a function $\phi \in C_{0}(I: X)$ such that $f(t)=h(t)+\phi(t)$ for all $t \in I$. This is equivalent to saying that, for every $\epsilon>0$, we can find numbers $l>0$ and $M>0$ such that every subinterval of $I^{\prime}$ of length $l$ contains, at least, one number $\tau$ such that $\|f(t+\tau)-f(t)\| \leq \epsilon$ provided $|t|,|t+\tau| \geq M$.

Following Haraux and Souplet [16], we say that the function $f(\cdot)$ is uniformly recurrent if and only if there exists a strictly increasing sequence $\left(\alpha_{n}\right)$ of positive real numbers such that $\lim _{n \rightarrow+\infty} \alpha_{n}=$ $+\infty$ and

$$
\lim _{n \rightarrow \infty} \sup _{t \in \mathbb{R}}\left\|f\left(t+\alpha_{n}\right)-f(t)\right\|=0 .
$$

It is well known that any almost periodic function is uniformly recurrent, while the converse statement is not true in general. The function $f: I \rightarrow X$ is said to be asymptotically uniformly recurrent if and only if there exist a uniformly recurrent function $h: I \rightarrow X$ and a function $\phi \in C_{0}(I: X)$ such that $f(t)=h(t)+\phi(t)$ for all $t \in I$. The classes of uniformly recurrent functions and asymptotically uniformly recurrent functions will be denoted by $U R(I: X)$ and $A U R(I: X)$, respectively.

Let $1 \leq p<\infty$. Then, we say that a function $f \in L_{l o c}^{p}(I: X)$ is Stepanov $p$-bounded if and only if

$$
\|f\|_{S^{p}}:=\sup _{t \in I}\left(\int_{t}^{t+1}\|f(s)\|^{p} d s\right)^{1 / p}=\sup _{t \in I}\left(\int_{0}^{1}\|f(s+t)\|^{p} d s\right)^{1 / p}<\infty .
$$

Endowed with the above norm, the space $L_{S}^{p}(I: X)$ consisting of all $S^{p}$-bounded functions becomes a Banach space. A function $f \in L_{S}^{p}(I: X)$ is said to be Stepanov $p$-almost periodic if and only if the function $\hat{f}: I \rightarrow L^{p}([0,1]: X)$, defined by

$$
\hat{f}(t)(s):=f(t+s), \quad t \in I, s \in[0,1],
$$

is almost periodic, while the function $f \in L_{l o c}^{p}(I: X)$ is said to be Stepanov $p$-uniformly recurrent if and only if the function $\hat{f}: I \rightarrow L^{p}([0,1]: X)$ is uniformly recurrent. A function $f \in L_{l o c}^{p}(I: X)$ is said to be asymptotically Stepanov $p$-uniformly recurrent if and only if there exist a Stepanov 
$p$-uniformly recurrent function $h(\cdot)$ and a function $q \in L_{S}^{p}(I: X)$ such that $f(t)=h(t)+q(t), t \in I$ and $\hat{q} \in C_{0}\left(I: L^{p}([0,1]: X)\right)$.

Let $\omega \in I$. Then, we say that a bounded continuous function $f: I \rightarrow X$ is $S$-asymptotically $\omega$-periodic if and only if $\lim _{|t| \rightarrow \infty}\|f(t+\omega)-f(t)\|=0$. Denote by $S A P_{\omega}(I: X)$ the space consisting of all such functions (see Henríquez et al. [17] for case $I=\mathbb{R}$ and Kostić [14] for case $I=[0, \infty)$ ). A Stepanov $p$-bounded function $f(\cdot)$ is said to be Stepanov $p$-asymptotically $\omega$-periodic if and only if

$$
\lim _{|t| \rightarrow \infty} \int_{t}^{t+1}\|f(s+\omega)-f(s)\|^{p} d s=0 .
$$

The class of quasi-asymptotically almost periodic functions extends the classes of asymptotically almost periodic functions and $S$-asymptotically $\omega$-periodic functions (a similar statement holds for the corresponding Stepanov classes).

Definition 1. It is said that a bounded continuous function $f: I \rightarrow X$ is quasi-asymptotically almost periodic if and only if for each $\epsilon>0$ there exists a finite number $L(\epsilon)>0$ such that any interval $I^{\prime} \subseteq$ I of length $L(\epsilon)$ contains at least one number $\tau \in I^{\prime}$ satisfying that there exists a finite number $M(\epsilon, \tau)>0$ such that

$$
\|f(t+\tau)-f(t)\| \leq \epsilon, \text { provided } t \in I \text { and }|t| \geq M(\epsilon, \tau) .
$$

Denote by $Q-A A P(I: X)$ the set consisting of all quasi-asymptotically almost periodic functions from I into X.

Definition 2. Let $f \in L_{S}^{p}(I: X)$. Then, it is said $f(\cdot)$ is Stepanov p-quasi-asymptotically almost periodic if and only if for each $\epsilon>0$ there exists a finite number $L(\epsilon)>0$ such that any interval $I^{\prime} \subseteq I$ of length $L(\epsilon)$ contains at least one number $\tau \in I^{\prime}$ satisfying that there exists a finite number $M(\epsilon, \tau)>0$ such that

$$
\int_{t}^{t+1}\|f(s+\tau)-f(s)\|^{p} d s \leq \epsilon^{p}, \text { provided } t \in I \text { and }|t| \geq M(\epsilon, \tau) .
$$

Denote by $S^{p} Q-A A P(I: X)$ the set consisting of all Stepanov p-quasi-asymptotically almost periodic functions from I into $X$.

\subsection{Lebesgue Spaces with Variable Exponents $L^{p(x)}$}

Let $\varnothing \neq \Omega \subseteq \mathbb{R}$ be a nonempty subset and let $M(\Omega: X)$ stand for the collection of all measurable functions $f: \Omega \rightarrow X ; M(\Omega):=M(\Omega: \mathbb{R})$. Furthermore, $\mathcal{P}(\Omega)$ denotes the vector space of all Lebesgue measurable functions $p: \Omega \rightarrow[1, \infty]$. For any $p \in \mathcal{P}(\Omega)$ and $f \in M(\Omega: X)$, set

$$
\varphi_{p(x)}(t):=\left\{\begin{array}{l}
t^{p(x)}, \quad t \geq 0,1 \leq p(x)<\infty \\
0, \quad 0 \leq t \leq 1, p(x)=\infty \\
\infty, \quad t>1, \quad p(x)=\infty
\end{array}\right.
$$

and

$$
\rho(f):=\int_{\Omega} \varphi_{p(x)}(\|f(x)\|) d x .
$$

We define the Lebesgue space $L^{p(x)}(\Omega: X)$ with variable exponent as follows:

$$
L^{p(x)}(\Omega: X):=\left\{f \in M(\Omega: X): \lim _{\lambda \rightarrow 0+} \rho(\lambda f)=0\right\}
$$


equivalently,

$$
L^{p(x)}(\Omega: X)=\{f \in M(\Omega: X): \text { there exists } \lambda>0 \text { such that } \rho(\lambda f)<\infty\} .
$$

For every $u \in L^{p(x)}(\Omega: X)$, we introduce the Luxemburg norm of $u(\cdot)$ by

$$
\|u\|_{p(x)}:=\|u\|_{L^{p(x)}(\Omega: X)}:=\inf \{\lambda>0: \rho(f / \lambda) \leq 1\} .
$$

Equipped with the above norm, the space $L^{p(x)}(\Omega: X)$ becomes a Banach space, coinciding with the usual Lebesgue space $L^{p}(\Omega: X)$ in the case that $p(x)=p \geq 1$ is a constant function. For any $p \in M(\Omega)$, we set

$$
p^{-}:=\operatorname{essinf}_{x \in \Omega} p(x) \text { and } p^{+}:=\operatorname{esssup}_{x \in \Omega} p(x) .
$$

Define

$$
C_{+}(\Omega):=\left\{p \in M(\Omega): 1<p^{-} \leq p(x) \leq p^{+}<\infty \text { for a.e. } x \in \Omega\right\}
$$

and

$$
D_{+}(\Omega):=\left\{p \in M(\Omega): 1 \leq p^{-} \leq p(x) \leq p^{+}<\infty \text { for a.e. } x \in \Omega\right\} .
$$

If $p \in D_{+}([0,1])$, then we know that

$$
L^{p(x)}(\Omega: X)=\{f \in M(\Omega: X): \text { for all } \lambda>0 \text { we have } \rho(\lambda f)<\infty\} .
$$

We will use the following lemma (see, e.g., Reference [18], (Lemma 3.2.20, (3.2.22); Corollary 3.3.4; p. 77) for the scalar-valued case):

Lemma 1. (i) (The Hölder inequality) Let $p, q, r \in \mathcal{P}(\Omega)$ such that

$$
\frac{1}{q(x)}=\frac{1}{p(x)}+\frac{1}{r(x)}, \quad x \in \Omega
$$

Then, for every $u \in L^{p(x)}(\Omega: X)$ and $v \in L^{r(x)}(\Omega)$, we have $u v \in L^{q(x)}(\Omega: X)$ and

$$
\|u v\|_{q(x)} \leq 2\|u\|_{p(x)}\|v\|_{r(x)} .
$$

(ii) Let $\Omega$ be of a finite Lebesgue's measure $m(\Omega)<\infty$, and let $p, q \in \mathcal{P}(\Omega)$ such $q \leq p$ a.e. on $\Omega$. Then, $L^{p(x)}(\Omega: X)$ is continuously embedded in $L^{q(x)}(\Omega: X)$, with the constant of embedding less than or equal to $2(1+m(\Omega))$.

(iii) Let $f \in L^{p(x)}(\Omega: X), g \in M(\Omega: X)$ and $0 \leq\|g\| \leq\|f\|$ a.e. on $\Omega$. Then, $g \in L^{p(x)}(\Omega: X)$ and $\|g\|_{p(x)} \leq\|f\|_{p(x)}$.

We will use the following fact. If $p \in \mathcal{P}(I), a, b, c \in I, a<b<c$ and $f \in L^{p(x)}[a, c]$, then $f \in L^{p(x)}[a, b], f \in L^{p(x)}[b, c]$ and

$$
\|f\|_{L^{p(x)}[a, c]} \leq\|f\|_{L^{p(x)}[a, b]}+\|f\|_{L^{p(x)}[b, c]} .
$$

For furher information concerning the Lebesgue spaces with variable exponents $L^{p(x)}$, we refer the reader to Reference $[8,9,18-20]$.

\section{Generalized Weyl Uniform Recurrence in Lebesgue Spaces with Variable Exponents $L^{p(x)}$}

Throughout this section, we use the following conditions:

$(A): \quad I=\mathbb{R}$ or $I=[0, \infty), \phi:[0, \infty) \rightarrow[0, \infty), p \in \mathcal{P}(I)$ and $F:(0, \infty) \times I \rightarrow(0, \infty)$. 
(B): The same as (A) with the assumption $p \in \mathcal{P}(I)$ replaced by $p \in \mathcal{P}([0,1])$ therein.

We will first extend the notion introduced in Reference [12] (Definitions 2.1-2.3):

Definition 3. Suppose that condition (A) holds, $f: I \rightarrow X$, and $\phi(\|f(\cdot+\tau)-f(\cdot)\|) \in L^{p(x)}(K)$ for any $\tau \in I$ and any compact subset $K$ of $I$.

(i) It is said that the function $f(\cdot)$ is equi-Weyl- $(p(x), \phi, F)$-uniformly recurrent, $f \in e-W_{u r}^{(p(x), \phi, F)}(I: X)$ for short, if and only if we can find two sequences $\left(l_{n}\right)$ and $\left(\alpha_{n}\right)$ of positive real numbers such that $\lim _{n \rightarrow+\infty} \alpha_{n}=+\infty$ and

$$
\lim _{n \rightarrow+\infty} \sup _{t \in I}\left[F\left(l_{n}, t\right)\left[\phi\left(\left\|f\left(\cdot+\alpha_{n}\right)-f(\cdot)\right\|\right)_{L^{p(\cdot)}\left[t, t+l_{n}\right]}\right]\right]=0 .
$$

(ii) It is said that the function $f(\cdot)$ is Weyl- $(p(x), \phi, F)$-uniformly recurrent, $f \in W_{u r}^{(p(x), \phi, F)}(I: X)$ for short, if and only if we can find a sequence $\left(\alpha_{n}\right)$ of positive real numbers such that $\lim _{n \rightarrow+\infty} \alpha_{n}=+\infty$ and

$$
\lim _{n \rightarrow+\infty} \limsup _{l \rightarrow \infty} \sup _{t \in I}\left[F(l, t)\left[\phi\left(\left\|f\left(\cdot+\alpha_{n}\right)-f(\cdot)\right\|\right)_{L^{p(\cdot)}[t, t+l]}\right]\right]=0 .
$$

Definition 4. Suppose that condition (A) holds, $f: I \rightarrow X$ and $\|f(\cdot+\tau)-f(\cdot)\| \in L^{p(x)}(K)$ for any $\tau \in I$ and any compact subset $K$ of $I$.

(i) It is said that the function $f(\cdot)$ is equi-Weyl- $(p(x), \phi, F)_{1}$-uniformly recurrent, $f \in e-W_{u r}^{(p(x), \phi, F)_{1}}(I$ : $X)$ for short, if and only if we can find two sequences $\left(l_{n}\right)$ and $\left(\alpha_{n}\right)$ of positive real numbers such that $\lim _{n \rightarrow+\infty} \alpha_{n}=+\infty$ and

$$
\lim _{n \rightarrow+\infty} \sup _{t \in I}\left[F\left(l_{n}, t\right) \phi\left[\left(\left\|f\left(\cdot+\alpha_{n}\right)-f(\cdot)\right\|\right)_{L^{p(\cdot)}\left[t, t+l_{n}\right]}\right]\right]=0 .
$$

(ii) It is said that the function $f(\cdot)$ is Weyl- $(p(x), \phi, F)_{1}$-uniformly recurrent, $f \in W_{u r}^{(p(x), \phi, F)_{1}}(I: X)$ for short, if and only if we can find a sequence ( $\left.\alpha_{n}\right)$ of positive real numbers such that $\lim _{n \rightarrow+\infty} \alpha_{n}=+\infty$ and

$$
\lim _{n \rightarrow+\infty} \limsup _{l \rightarrow \infty} \sup _{t \in I}\left[F(l, t) \phi\left[\left(\left\|f\left(\cdot+\alpha_{n}\right)-f(\cdot)\right\|\right)_{L^{p(\cdot)}[t, t+l]}\right]\right]=0 .
$$

Definition 5. Suppose that condition (A) holds, $f: I \rightarrow X$ and $\|f(\cdot+\tau)-f(\cdot)\| \in L^{p(x)}(K)$ for any $\tau \in I$ and any compact subset $K$ of $I$.

(i) It is said that the function $f(\cdot)$ is equi-Weyl- $(p(x), \phi, F)_{2}$-uniformly recurrent, $f \in e-W_{u r}^{(p(x), \phi, F)_{2}}(I$ : $X)$ for short, if and only if we can find two sequences $\left(l_{n}\right)$ and $\left(\alpha_{n}\right)$ of positive real numbers such that $\lim _{n \rightarrow+\infty} \alpha_{n}=+\infty$ and

$$
\lim _{n \rightarrow+\infty} \sup _{t \in I} \phi\left[F\left(l_{n}, t\right)\left[\left(\left\|f\left(\cdot+\alpha_{n}\right)-f(\cdot)\right\|\right)_{L^{p(\cdot)}\left[t, t+l_{n}\right]}\right]\right]=0 .
$$

(ii) It is said that the function $f(\cdot)$ is Weyl- $(p(x), \phi, F)_{2}$-uniformly recurrent, $f \in W_{u r}^{(p(x), \phi, F)_{2}}(I: X)$ for short, if and only if we can find a sequence $\left(\alpha_{n}\right)$ of positive real numbers such that $\lim _{n \rightarrow+\infty} \alpha_{n}=+\infty$ and

$$
\lim _{n \rightarrow+\infty} \limsup _{l \rightarrow \infty} \sup _{t \in I} \phi\left[F(l, t)\left[\left(\left\|f\left(\cdot+\alpha_{n}\right)-f(\cdot)\right\|\right)_{L^{p(\cdot)}[t, t+l]}\right]\right]=0 .
$$


Before we go any further, we would like to that the above definitions are incapable of being compared to each other: For example, in Definition 3, we calculate the value of $\phi\left(\| f\left(\cdot+\alpha_{n}\right)-\right.$ $f(\cdot) \|)_{L^{p(\cdot)}\left[t, t+l_{n}\right]}$, while, in Definition 4, we first calculate the value of $\left\|f\left(\cdot+\alpha_{n}\right)-f(\cdot)\right\|_{L^{p(\cdot)}\left[t, t+l_{n}\right]}$, and, after that, we apply the function $\phi(\cdot)$.

It is clear that the class of (equi-)Weyl- $(p(x), \phi, F)$-uniformly recurrent functions, resp. (equi-)Weyl- $(p(x), \phi, F)_{i}$-uniformly recurrent functions, extends the class of (equi-)Weyl- $(p(x), \phi, F)$ almost periodic functions, resp. (equi-)Weyl- $(p(x), \phi, F)_{i}$-almost periodic functions $(i=1,2)$. Case $p(x) \equiv p, \phi(x) \equiv x$ and $F(l, t)=l^{(-1) / p}$ is the most indicative, when we say that the function $f(\cdot)$ is (equi-)Weyl- $p$-uniformly recurrent. The class of (equi-)Weyl- $p$-uniformly recurrent functions has not been considered elsewhere by now.

Let us recall that any equi-Weyl- $p$-almost periodic function needs to be Weyl $p$-almost periodic, while the converse statement does not hold in general. In Reference [12], we showed that an equi-Weyl- $(p, \phi, \psi)$-almost periodic function, resp. equi-Weyl- $(p, \phi, \psi)_{i}$-almost periodic function, does not need to be Weyl- $(p, \phi, \psi)$-almost periodic, resp. Weyl- $(p, \phi, \psi)_{i}$-almost periodic $(i=1,2)$. This statement continues to hold for generalized uniformly recurrent functions introduced above. For example, the use of same arguments as in Reference [12] shows that any continuous Stepanov $p$-almost periodic function $f(\cdot)$ which is not periodic cannot be Weyl- $(p, x, 1)$-uniformly recurrent, while it is always equi-Weyl- $(p, x, 1)$-almost periodic.

Example 1. If $X$ does not contain an isomorphic copy of the sequence space $c_{0}, \phi(x)=x$ and $F(l, t) \equiv F(t)$, where $\lim _{t \rightarrow+\infty} F(t)=+\infty$, then there does not exist a non-periodic trigonometric polynomial $f(\cdot)$ and function $p \in \mathcal{P}(\mathbb{R})$ such that $f \in e-W_{\text {ur }}^{(p, x, F)}(\mathbb{R}: X)$. This can be verified based on the argumentation contained in Reference [12] (example 2.4 (iii)).

Furthermore, the statement of Reference [12] (Proposition 2.5) and the conclusions established in Reference [12] (Remark 2.6) can be reformulated for the introduced classes of generalized Weyl uniformly recurrent functions. In order to ensure the translation invariance of generalized Weyl spaces of uniformly recurrent functions with variable exponents, we will follow a slightly different approach based on the idea from Reference [10] (in the subsequent definitions, we use condition (B) so that they cannot be so easily connected with Definitions 3-5):

Definition 6. Suppose that condition (B) holds, $f: I \rightarrow X$ and $\phi(\|f(\cdot l+t+\tau)-f(t+\cdot l)\|) \in L^{p(x)}([0,1])$ for any $\tau \in I, t \in I$ and $l>0$.

(i) It is said that the function $f(\cdot)$ is equi-Weyl- $[p(x), \phi, F]$-uniformly recurrent, $f \in e-W_{u r}^{[p(x), \phi, F]}(I: X)$ for short, if and only if we can find two sequences $\left(l_{n}\right)$ and $\left(\alpha_{n}\right)$ of positive real numbers such that $\lim _{n \rightarrow+\infty} \alpha_{n}=+\infty$ and

$$
\lim _{n \rightarrow+\infty} \sup _{t \in I}\left[F\left(l_{n}, t\right)\left[\phi\left(\left\|f\left(\cdot l_{n}+t+\alpha_{n}\right)-f\left(t+\cdot l_{n}\right)\right\|\right)_{L^{p(\cdot)}[0,1]}\right]\right]=0 .
$$

(ii) It is said that the function $f(\cdot)$ is Weyl- $[p(x), \phi, F]$-uniformly recurrent, $f \in W_{u r}^{[p(x), \phi, F]}(I: X)$ for short, if and only if we can find a sequence $\left(\alpha_{n}\right)$ of positive real numbers such that $\lim _{n \rightarrow+\infty} \alpha_{n}=+\infty$ and

$$
\lim _{n \rightarrow+\infty} \limsup _{l \rightarrow \infty} \sup _{t \in I}\left[F(l, t)\left[\phi\left(\left\|f\left(\cdot l+t+\alpha_{n}\right)-f(t+\cdot l)\right\|\right)_{L^{p(\cdot)}[0,1]}\right]\right]=0 .
$$

Definition 7. Suppose that condition (B) holds, $f: I \rightarrow X$ and $\|f(\cdot l+t+\tau)-f(t+\cdot l)\| \in L^{p(x)}([0,1])$ for any $\tau \in I, t \in I$ and $l>0$. 
(i) It is said that the function $f(\cdot)$ is equi-Weyl- $[p(x), \phi, F]_{1}$-uniformly recurrent, $f \in e-W_{u r}^{[p(x), \phi, F]_{1}}(I: X)$ for short, if and only if we can find two sequences $\left(l_{n}\right)$ and $\left(\alpha_{n}\right)$ of positive real numbers such that $\lim _{n \rightarrow+\infty} \alpha_{n}=+\infty$ and

$$
\lim _{n \rightarrow+\infty} \sup _{t \in I}\left[F\left(l_{n}, t\right) \phi\left[\left(\left\|f\left(\cdot l_{n}+t+\alpha_{n}\right)-f\left(t+\cdot l_{n}\right)\right\|\right)_{L^{p(\cdot)}[0,1]}\right]\right]=0 .
$$

(ii) It is said that the function $f(\cdot)$ is Weyl- $[p(x), \phi, F]_{2}$-uniformly recurrent, $f \in W_{u r}^{[p(x), \phi, F]_{2}}(I: X)$ for short, if and only if we can find a sequence $\left(\alpha_{n}\right)$ of positive real numbers such that $\lim _{n \rightarrow+\infty} \alpha_{n}=+\infty$ and

$$
\lim _{n \rightarrow+\infty} \limsup _{l \rightarrow \infty} \sup _{t \in I}\left[F(l, t) \phi\left[\left(\left\|f\left(\cdot l+t+\alpha_{n}\right)-f(t+\cdot l)\right\|\right)_{L^{p(\cdot)}[0,1]}\right]\right]=0 .
$$

Definition 8. Suppose that condition (B) holds, $f: I \rightarrow X$ and $\|f(\cdot l+t+\tau)-f(t+\cdot l)\| \in L^{p(x)}([0,1])$ for any $\tau \in I, t \in I$ and $l>0$.

(i) It is said that the function $f(\cdot)$ is equi-Weyl- $[p(x), \phi, F]_{2}$-uniformly recurrent, $f \in e-W_{u r}^{[p(x), \phi, F]_{2}}(I$ : $X)$ for short, if and only we can find two sequences $\left(l_{n}\right)$ and $\left(\alpha_{n}\right)$ of positive real numbers such that $\lim _{n \rightarrow+\infty} \alpha_{n}=+\infty$ and

$$
\lim _{n \rightarrow+\infty} \sup _{t \in I} \phi\left[F\left(l_{n}, t\right)\left[\left(\left\|f\left(\cdot l_{n}+t+\alpha_{n}\right)-f\left(t+\cdot l_{n}\right)\right\|\right)_{L^{p(\cdot)}[0,1]}\right]\right]=0 .
$$

(ii) It is said that the function $f(\cdot)$ is Weyl- $[p(x), \phi, F]_{2}$-uniformly recurrent, $f \in W_{u r}^{[p(x), \phi, F]_{2}}(I: X)$ for short, if and only if we can find a sequence ( $\left.\alpha_{n}\right)$ of positive real numbers such that $\lim _{n \rightarrow+\infty} \alpha_{n}=+\infty$ and

$$
\lim _{n \rightarrow+\infty} \limsup _{l \rightarrow \infty} \sup _{t \in I} \phi\left[F(l, t)\left[\left(\left\|f\left(\cdot l+t+\alpha_{n}\right)-f(t+\cdot l)\right\|\right)_{L^{p(\cdot)}[0,1]}\right]\right]=0 .
$$

The statement of Reference [12] (Proposition 2.11) and the conclusions established in Reference [12] (Remark 2.10) can be reformulated for generalized Weyl uniformly recurrent functions introduced in the above three definitions. In Reference [12] (Theorem 4.1, Theorem 4.4), we analyzed the invariance of (equi-)Weyl- $(p, \phi, F)$-almost periodicity and (equi-)Weyl- $[p, \phi, F]$-almost periodicity under the actions of infinite convolution products; also see Reference [12] (Theorems 4.6 and 4.7), resp. Reference [12] (Theorems 4.9 and 4.10), where we analyzed the invariance of (equi-)Weyl- $(p, \phi, F)_{1}$-almost periodicity and (equi-)Weyl- $[p, \phi, F]_{1}$-almost periodicity, resp. (equi-)Weyl- $(p, \phi, F)_{2}$-almost periodicity and (equi-)Weyl- $[p, \phi, F]_{2}$-almost periodicity, under the actions of infinite convolution products. Furthermore, in Reference [12] (Propositions 4.12 and 4.13), we examined the convolution invariance of the above-mentioned types of generalized Weyl almost periodicity with variable exponents. All these statements can be straightforwardly reformulated for generalized Weyl uniformly recurrent functions introduced in this section; we leave readers to make this precise.

\section{Quasi-Asymptotically Uniformly Recurrent Type Functions with Variable Exponents}

In the following definition, we will extend the notion of quasi-asymptotical almost periodicity introduced in Reference [14] (Definition 2.1) (also see Reference [14], Remark 2.2): 
Definition 9. We say that a continuous function $f: I \rightarrow X$ is quasi-asymptotically uniformly recurrent if and only if there exists a strictly increasing sequence $\left(\alpha_{n}\right)$ of positive real numbers tending to plus infinity and a sequence $\left(M_{n}\right)$ of positive real numbers such that

$$
\lim _{n \rightarrow+\infty} \sup _{|t| \geq M_{n}}\left\|f\left(t+\alpha_{n}\right)-f(t)\right\|=0
$$

Denote by $Q-A U R(I: X)$ the set consisting of all quasi-asymptotically uniformly recurrent functions from I into $X$.

Besides the class $Q-A A P(I: X)$, it is expected that the class of quasi-asymptotically uniformly recurrent functions extends the class of asymptotically uniformly recurrent functions, as well. For completeness, we will include all details of the proof of the following proposition:

Proposition 1. Suppose that $f: I \rightarrow X$ is asymptotically uniformly recurrent. Then, $f(\cdot)$ is quasi-asymptotically uniformly recurrent.

Proof. Let $h \in U R(I: X), q \in C_{0}(I: X)$ and $f=h+q$. By our assumption, we have the existence of a strictly increasing sequence $\left(\alpha_{n}\right)$ of positive real numbers tending to plus infinity such that (2) holds with the function $f(\cdot)$ replaced therein with the function $h(\cdot)$. Let $n \in \mathbb{N}$ be fixed. Then, we can find a sufficiently large real number $M_{n}^{\prime}>0$ and a sufficiently large integer $n_{0} \in \mathbb{N}$ such that $\|q(t)\| \leq 1 / n$ for $|t| \geq M_{n}^{\prime}$ and $\left\|h\left(t+\alpha_{n}\right)-h(t)\right\| \leq 1 / n, n \geq n_{0}$. Then, for every $t \in \mathbb{R}$ such that $|t| \geq M_{n}:=M_{n}^{\prime}+\alpha_{n}$, we have $|t|,\left|t+\alpha_{n}\right| \geq M_{n}^{\prime}$ and

$$
\left\|\left[h\left(t+\alpha_{n}\right)-h(t)\right]+\left[q\left(t+\alpha_{n}\right)-q(t)\right]\right\| \leq \frac{1}{n}+\left\|q\left(t+\alpha_{n}\right)-q(t)\right\| \leq \frac{2}{n}, n \geq n_{0} .
$$

This simply implies the required assertion.

Applying the same arguments, we can deduce the following

Proposition 2. Suppose that $f: I \rightarrow X$ is quasi-asymptotically uniformly recurrent and $q \in C_{0}(I: X)$. Then, $(f+q)(\cdot)$ is likewise quasi-asymptotically uniformly recurrent.

The proof of following proposition is simple and can be omitted, as well:

Proposition 3. Suppose that $I=\mathbb{R}$ and $f: I \rightarrow X$. Then, $f(\cdot)$ is quasi-asymptotically uniformly recurrent (quasi-asymptotically almost periodic, S-asymptotically $\omega$-periodic) if and only if $\breve{f}(\cdot)$ is quasi-asymptotically uniformly recurrent (quasi-asymptotically almost periodic, S-asymptotically $\omega$-periodic).

If $f \in Q-A U R(\mathbb{R}: X)$ and $\varphi \in L^{1}(\mathbb{R})$ has a compact support, then it can be easily seen that the convolution $\varphi \times f(\cdot):=\int_{\mathbb{R}} \varphi(\cdot-y) f(y) d y$ belongs to the class $Q-A U R(\mathbb{R}: X)$. Furthermore, any quasi-asymptotically almost periodic function is bounded by definition, and this is no longer true for quasi-asymptotically uniformly recurrent functions. In connection with this, we would like to present the following illustrative example:

Example 2. (Reference $[16,21])$ Set

$$
f(t):=\sum_{n=1}^{\infty} \frac{1}{n} \sin ^{2}\left(\frac{t}{2^{n}}\right), \quad t \in \mathbb{R} .
$$

We know that, for each real number $c>0$, the function $h(t):=\min (c, f(t)), t \in \mathbb{R}$ is bounded uniformly continuous, uniformly recurrent, and not (Stepanov) p-quasi-asymptotically almost periodic $(p \geq 1)$. On the other hand, Proposition 1 shows that the function $h(\cdot)$ is quasi-asymptotically uniformly recurrent. 
Furthermore, if $f \in C^{1}(I: X)$ and $f^{\prime} \in C_{0}(I: X)$, then the Lagrange mean value theorem implies that the function $f(\cdot)$ is quasi-asymptotically uniformly recurrent. In particular, the function $f(t):=$ $\ln (1+t), t \geq 0$ is quasi-asymptotically uniformly recurrent; on the other hand, it can be simply verified that the function $f(\cdot)$ is not asymptotically uniformly recurrent. The notion of quasi-asymptotical uniform anti-recurrence can be also introduced and analyzed (also see Reference [14] (Example 2.3, Remark 2.4)).

Example 3. The function $f:[0, \infty) \rightarrow \mathbb{R}$ given by $f(t):=\sin (\ln (1+t)), t \geq 0$ is quasi-asymptotically almost periodic but not asymptotically almost periodic (see Reference [14,22] (Example 4.1, Theorem 4.2)). Now we will prove that this function cannot be asymptotically uniformly recurrent. Suppose the contrary, and fix a sufficiently small number $\epsilon>0$. Then, an elementary argumentation shows that there exists a strictly increasing sequence $\left(\alpha_{n}\right)$ of positive real numbers tending to plus infinity and a number $t_{0}(\epsilon)>0$ such that $\left|\sin \left(\ln \left(t+\alpha_{n}\right)\right)-\sin (\ln t)\right| \leq 2 \epsilon$ for all $t \geq t_{0}(\epsilon)$ and $n \in \mathbb{N}$. Hence,

$$
\left|\sin \frac{\ln \left(1+\left(\alpha_{n} / t\right)\right)}{2} \cos \frac{\ln \left(t\left(t+\alpha_{n}\right)\right)}{2}\right| \leq \epsilon, \quad t \geq t_{0}(\epsilon), n \in \mathbb{N} .
$$

Let $n_{0}(\epsilon) \in \mathbb{N}$ be such that $\alpha_{n} \geq t_{0}(\epsilon)$ for $n \geq n_{0}(\epsilon)$. Plugging $t=k \alpha_{n}$, where $1 \leq k \leq 5$, the above estimate simply implies that there exists a finite constant $c>0$ such that

$$
\left|\cos \frac{\ln \left(a \alpha_{n}^{2}\right)}{2}\right| \leq c \epsilon, \quad 2 \leq a \leq 30, n \geq n_{0}(\epsilon) .
$$

Then, we get the existence of a real number $c_{\epsilon}>0$ such that $\lim _{\epsilon \rightarrow 0+} c_{\epsilon}=0$ and

$$
\operatorname{dist}\left(a \alpha_{n}^{2},\left\{\exp ((2 k+1) \pi): k \in \mathbb{N}_{0}\right\}\right) \leq e^{2 c_{\epsilon}}, \quad 2 \leq a \leq 30, n \geq n_{0}(\epsilon) .
$$

It can be simply verified that this estimate cannot be satisfied simultaneously for $a=2$ and $a=e^{\pi}$, which yields a contradiction.

In Reference [14] (Theorem 2.5), we proved that any asymptotically almost automorphic function which is also quasi-asymptotically almost periodic is always asymptotically almost periodic. The arguments contained in the proof of the above-mentioned theorem also show that any asymptotically uniformly recurrent function which is quasi-asymptotically almost periodic needs to be asymptotically almost periodic (see Reference [21], Theorem 2.13 (i)), as well as that the following result holds true:

Theorem 1. Let $\mathrm{F}(I: X)$ be any space of functions $h: I \rightarrow X$ satisfying that, for each $\tau \in I$, the supremum formula holds for the function $h(\cdot+\tau)-h(\cdot)$, that is

$$
\sup _{t \in I}\|h(\cdot+\tau)-h(\cdot)\|=\sup _{t \in I,|t| \geq a}\|h(\cdot+\tau)-h(\cdot)\|, \quad a \in I .
$$

Then, we have:

(i) $\left[\mathrm{F}(I: X)+C_{0}(I: X)\right] \cap Q-\operatorname{AUR}(I: X) \subseteq \operatorname{AUR}(I: X)$.

(ii) $\mathrm{F}(I: X) \cap Q-A U R(I: X) \subseteq U R(I: X)$.

Proof. We will include the main details of the proof for the sake of completeness. Let $h \in \mathrm{F}(I: X)$, $q \in C_{0}(I: X)$ and $f=h+q \in Q-A U R(I: X)$. By our assumptions, there exists a strictly increasing sequence $\left(\alpha_{n}\right)$ of positive real numbers tending to plus infinity and a sequence $\left(M_{n}\right)$ of positive real numbers such that, for every integer $n \in \mathbb{N}$, there exists an integer $n_{0} \in \mathbb{N}$ with 


$$
\left\|\left[h\left(t+\alpha_{n}\right)-h(t)\right]+\left[q\left(t+\alpha_{n}\right)-q(t)\right]\right\| \leq 1 / n, \text { for } t \in I,|t| \geq M_{n}, n \geq n_{0} .
$$

Let $n \in \mathbb{N}$ be fixed. Since $q \in C_{0}(I: X)$, we have that there exists a finite number $M_{n}^{\prime} \geq M_{n}$ such that

$$
\left\|h\left(t+\alpha_{n}\right)-h(t)\right\| \leq 2 / n, \text { provided } t \in I \text { and }|t| \geq M_{n}^{\prime}, n \geq n_{0} .
$$

Define the function $H_{n}: I \rightarrow X$ by $H_{n}(t):=h\left(t+\alpha_{n}\right)-h(t), t \in I$. Since the supremum formula holds for the function $H_{n}(\cdot)$, we get

$$
\sup _{t \in I}\left\|H_{n}(t)\right\|=\sup _{t \geq M_{n}^{\prime}}\left\|H_{n}(t)\right\| \leq 2 / n
$$

Hence, $\lim _{n \rightarrow+\infty} \sup _{t \in I}\left\|h\left(t+\alpha_{n}\right)-h(t)\right\|=0$ and $h(\cdot)$ is thus uniformly recurrent, which immediately implies part (i). Part (ii) can be deduced similarly.

In the following illustrative application of Theorem 1 , we will consider the case in which $I=\mathbb{R}$ and $\mathrm{F}(I: X)=A A(I: X)$, the space of all almost automorphic functions from $I$ into $X$ (see Reference [5] for more details):

Example 4. The first example of a scalar-valued compactly almost automorphic function which is not almost periodic has been constructed by Fink (see [23], p. 521). Set $a_{n}:=\operatorname{sign}(\cos (n \pi \sqrt{2})), n \in \mathbb{Z}$ and define after that the function $f: \mathbb{R} \rightarrow \mathbb{R}$ by $f(t):=\alpha a_{n}+(1-\alpha) a_{n+1}$ if $t \in[n, n+1)$ for some integer $n \in \mathbb{Z}$ and $t=\alpha n+(1-\alpha)(n+1)$ for some number $\alpha \in(0,1]$. This function is compactly almost automorphic but not almost periodic; furthermore, in Reference [21], we proved that the function $f(\cdot)$ is not asymptotically uniformly recurrent. Using this fact and Theorem 1, it readily follows that the function $f(\cdot)$ is not quasi-asymptotically uniformly recurrent, as well.

\subsection{Stepanov Classes of Quasi-Asymptotically Uniformly Recurrent Type Functions}

Throughout this subsection, we use the following conditions:

$(A)_{S}: I=\mathbb{R}$ or $I=[0, \infty), \phi:[0, \infty) \rightarrow[0, \infty), p \in \mathcal{P}(I), \mathrm{F}: I \times(0, \infty) \times I \rightarrow(0, \infty), F: I \times \mathbb{N} \rightarrow$ $(0, \infty), \mathrm{F}: I \rightarrow(0, \infty)$ and $\omega \in I$.

$(B)_{S}$ : The same as $(A)_{S}$ with the assumption $p \in \mathcal{P}(I)$ replaced by $p \in \mathcal{P}([0,1])$ therein.

We will first introduce the Stepanov- $(p, \phi, F)$-classes of quasi-asymptotically uniformly recurrent functions and the Stepanov- $(p, \phi, F)_{i}$-classes of quasi-asymptotically uniformly recurrent functions, where $i=1,2$ and $p \in \mathcal{P}(I)$. In this approach, we may lose the information about the translation invariance of introduced spaces:

Definition 10. Let $(A)_{S}$ hold.

(i) A function $f: I \rightarrow X$ is called Stepanov- $(p, \phi, F)$-quasi-asymptotically almost periodic, resp. Stepanov- $(p, \phi, F)$-quasi-asymptotically uniformly recurrent, if and only if $\phi(\|f(\cdot+\tau)-f(\cdot)\|) \in$ $L^{p(\cdot)}(K)$ for any $\tau \in I$ and any compact set $K \subseteq I$, as well as for each $\epsilon>0$, there exists a finite number $L(\epsilon)>0$ such that any interval $I^{\prime} \subseteq I$ of length $L(\epsilon)$ contains at least one number $\tau \in I^{\prime}$ satisfying that there exists a finite number $M(\epsilon, \tau)>0$ such that

$$
\sup _{|t| \geq M(\epsilon, \tau)} F(t, \epsilon, \tau) \phi(\|f(\cdot+\tau)-f(\cdot)\|)_{L^{p(\cdot)}[t, t+1]} \leq \epsilon,
$$

resp. there exists a strictly increasing sequence $\left(\alpha_{n}\right)$ of positive real numbers tending to plus infinity and a sequence $\left(M_{n}\right)$ of positive real numbers such that 


$$
\lim _{n \rightarrow+\infty} \sup _{|t| \geq M_{n}} F(t, n) \phi\left(\left\|f\left(\cdot+\alpha_{n}\right)-f(\cdot)\right\|\right)_{L^{p(\cdot)}[t, t+1]}=0
$$

(ii) We say that a function $f: I \rightarrow X$ is Stepanov- $(p, \phi, F)$-asymptotically $\omega$-periodic if and only if $\phi(\| f(\cdot+$ $\omega)-f(\cdot) \|) \in L^{p(\cdot)}(K)$ for any compact set $K \subseteq I$ and

$$
\lim _{|t| \rightarrow \infty} F(t) \phi(\|f(\cdot+\omega)-f(\cdot)\|)_{L^{p(\cdot)}[t, t+1]}=0 .
$$

Definition 11. Let $(A)_{S}$ hold.

(i) A function $f: I \rightarrow X$ is called Stepanov- $(p, \phi, F)_{1}$-quasi-asymptotically almost periodic, resp. Stepanov- $(p, \phi, F)_{1}$-quasi-asymptotically uniformly recurrent, if and only if $\|f(\cdot+\tau)-f(\cdot)\| \in L^{p(\cdot)}(K)$ for any $\tau \in I$ and any compact set $K \subseteq I$, as well as for each $\epsilon>0$, there exists a finite number $L(\epsilon)>0$ such that any interval $I^{\prime} \subseteq$ I of length $L(\epsilon)$ contains at least one number $\tau \in I^{\prime}$ satisfying that there exists a finite number $M(\epsilon, \tau)>0$ such that

$$
\sup _{|t| \geq M(\epsilon, \tau)} F(t, \epsilon, \tau) \phi\left(\|f(\cdot+\tau)-f(\cdot)\|_{L^{p(\cdot)}[t, t+1]}\right) \leq \epsilon,
$$

resp. there exists a strictly increasing sequence $\left(\alpha_{n}\right)$ of positive real numbers tending to plus infinity and a sequence $\left(M_{n}\right)$ of positive real numbers such that

$$
\lim _{n \rightarrow+\infty} \sup _{|t| \geq M_{n}} F(t, n) \phi\left(\left\|f\left(\cdot+\alpha_{n}\right)-f(\cdot)\right\|_{L^{p(\cdot)}[t, t+1]}\right)=0 .
$$

(ii) We say that a function $f: I \rightarrow X$ is Stepanov- $(p, \phi, F)_{1}$-asymptotically $\omega$-periodic if and only if $\|f(\cdot+\omega)-f(\cdot)\| \in L^{p(\cdot)}(K)$ for any compact set $K \subseteq I$ and

$$
\lim _{|t| \rightarrow \infty} F(t) \phi\left(\|f(\cdot+\omega)-f(\cdot)\|_{L^{p(\cdot)}[t, t+1]}\right)=0 .
$$

Definition 12. Let $(A)_{S}$ hold.

(i) A function $f: I \rightarrow X$ is called Stepanov- $(p, \phi, F)_{2}$-quasi-asymptotically almost periodic, resp. Stepanov$(p, \phi, F)_{2}$-quasi-asymptotically uniformly recurrent, if and only if $\|f(\cdot+\tau)-f(\cdot)\| \in L^{p(\cdot)}(K)$ for any $\tau \in I$ and any compact set $K \subseteq I$, as well as for each $\epsilon>0$, there exists a finite number $L(\epsilon)>0$ such that any interval $I^{\prime} \subseteq I$ of length $L(\epsilon)$ contains at least one number $\tau \in I^{\prime}$ satisfying that there exists a finite number $M(\epsilon, \tau)>0$ such that

$$
\sup _{|t| \geq M(\epsilon, \tau)} \phi\left(F(t, \epsilon, \tau)\|f(\cdot+\tau)-f(\cdot)\|_{L^{p(\cdot)}[t, t+1]}\right) \leq \epsilon,
$$

resp. there exists a strictly increasing sequence $\left(\alpha_{n}\right)$ of positive real numbers tending to plus infinity and a sequence $\left(M_{n}\right)$ of positive real numbers such that

$$
\lim _{n \rightarrow+\infty} \sup _{|t| \geq M_{n}} \phi\left(F(t, n)\left\|f\left(\cdot+\alpha_{n}\right)-f(\cdot)\right\|_{L^{p(\cdot)}[t, t+1]}\right)=0 .
$$

(ii) Then, we say that a function $f: I \rightarrow X$ is Stepanov- $(p, \phi, F)_{2}$-asymptotically $\omega$-periodic if and only if $\|f(\cdot+\omega)-f(\cdot)\| \in L^{p(\cdot)}(K)$ for any compact set $K \subseteq I$ and

$$
\lim _{|t| \rightarrow \infty} \phi\left(F(t)\|f(\cdot+\omega)-f(\cdot)\|_{L^{p(\cdot)}[t, t+1]}\right)=0 \text {. }
$$


In the second approach, we will employ condition $(B)_{S}$ and therefore assume that $p \in \mathcal{P}([0,1])$. Using the substitution $\cdot \rightarrow \cdot+t$, the translation invariance of considered function spaces can be achieved (see e.g., Reference [12], Remark 2.10 (iii)):

Definition 13. Let $(B)_{S}$ hold.

(i) A function $f: I \rightarrow X$ is called Stepanov- $[p, \phi, F]$-quasi-asymptotically almost periodic, resp. Stepanov- $[p, \phi, F]$-quasi-asymptotically uniformly recurrent, if and only if $\phi(\|f(\cdot+t+\tau)-f(\cdot+t)\|) \in$ $L^{p(\cdot)}[0,1]$ for any $\tau, t \in I$, as well as for each $\epsilon>0$, there exists a finite number $L(\epsilon)>0$ such that any interval $I^{\prime} \subseteq I$ of length $L(\epsilon)$ contains at least one number $\tau \in I^{\prime}$ satisfying that there exists a finite number $M(\epsilon, \tau)>0$ such that

$$
\sup _{|t| \geq M(\epsilon, \tau)} F(t, \epsilon, \tau) \phi(\|f(\cdot+t+\tau)-f(\cdot+t)\|)_{L^{p(\cdot)}[0,1]} \leq \epsilon,
$$

resp. there exists a strictly increasing sequence $\left(\alpha_{n}\right)$ of positive real numbers tending to plus infinity and a sequence $\left(M_{n}\right)$ of positive real numbers such that

$$
\lim _{n \rightarrow+\infty} \sup _{|t| \geq M_{n}} F(t, n) \phi\left(\left\|f\left(\cdot+t+\alpha_{n}\right)-f(\cdot+t)\right\|\right)_{L^{p(\cdot)}[0,1]}=0 .
$$

(ii) Then, we say that a function $f: I \rightarrow X$ is Stepanov- $[p, \phi, F]$-asymptotically $\omega$-periodic if and only if $\phi(\|f(\cdot+t+\omega)-f(\cdot+t)\|) \in L^{p(\cdot)}[0,1]$ for any $t \in I$ and

$$
\lim _{|t| \rightarrow \infty} F(t) \phi(\|f(\cdot+t+\omega)-f(\cdot+t)\|)_{L^{p(\cdot)}[0,1]}=0
$$

Definition 14. Let $(B)_{S}$ hold.

(i) A function $f: I \rightarrow X$ is called Stepanov- $[p, \phi, F]_{1}-q u a s i$-asymptotically almost periodic, resp. Stepanov- $[p, \phi, F]_{1}$-quasi-asymptotically uniformly recurrent, if and only if $\|f(\cdot+t+\tau)-f(\cdot+t)\| \in$ $L^{p(\cdot)}[0,1]$ for any $\tau, t \in I$, as well as for each $\epsilon>0$, there exists a finite number $L(\epsilon)>0$ such that any interval $I^{\prime} \subseteq I$ of length $L(\epsilon)$ contains at least one number $\tau \in I^{\prime}$ satisfying that there exists a finite number $M(\epsilon, \tau)>0$ such that

$$
\sup _{|t| \geq M(\epsilon, \tau)} F(t, \epsilon, \tau) \phi\left(\|f(\cdot+t+\tau)-f(\cdot+t)\|_{L^{p(\cdot)}[0,1]}\right) \leq \epsilon,
$$

resp. there exists a strictly increasing sequence $\left(\alpha_{n}\right)$ of positive real numbers tending to plus infinity and a sequence $\left(M_{n}\right)$ of positive real numbers such that

$$
\lim _{n \rightarrow+\infty} \sup _{|t| \geq M_{n}} F(t, n) \phi\left(\left\|f\left(\cdot+t+\alpha_{n}\right)-f(\cdot+t)\right\|_{L^{p(\cdot)}[0,1]}\right)=0 .
$$

(ii) Then, we say that a function $f: I \rightarrow X$ is Stepanov- $[p, \phi, F]_{1}$-asymptotically $\omega$-periodic if and only if $\|f(\cdot+t+\omega)-f(\cdot+t)\| \in L^{p(\cdot)}[0,1]$ for any $t \in I$ and

$$
\lim _{|t| \rightarrow \infty} F(t) \phi\left(\|f(\cdot+t+\omega)-f(\cdot+t)\|_{L^{p(\cdot)}[0,1]}\right)=0 .
$$

Definition 15. Let $(B)_{S}$ hold.

(i) A function $f: I \rightarrow X$ is called Stepanov- $[p, \phi, F]_{2}-q u a s i$-asymptotically almost periodic, resp. Stepanov- $[p, \phi, F]_{2}$-quasi-asymptotically uniformly recurrent, if and only if $\|f(\cdot+t+\tau)-f(\cdot+t)\| \in$ $L^{p(\cdot)}[0,1]$ for any $\tau, t \in I$, as well as for each $\epsilon>0$, there exists a finite number $L(\epsilon)>0$ such that 
any interval $I^{\prime} \subseteq I$ of length $L(\epsilon)$ contains at least one number $\tau \in I^{\prime}$ satisfying that there exists a finite number $M(\epsilon, \tau)>0$ such that

$$
\sup _{|t| \geq M(\epsilon, \tau)} \phi\left(F(t, \epsilon, \tau)\|f(\cdot+t+\tau)-f(\cdot+t)\|_{L^{p(\cdot)}[0,1]}\right) \leq \epsilon
$$

resp. there exists a strictly increasing sequence $\left(\alpha_{n}\right)$ of positive real numbers tending to plus infinity and a sequence $\left(M_{n}\right)$ of positive real numbers such that

$$
\lim _{n \rightarrow+\infty} \sup _{|t| \geq M_{n}} \phi\left(F(t, n)\left\|f\left(\cdot+t+\alpha_{n}\right)-f(\cdot+t)\right\|_{L^{p(\cdot)}[0,1]}\right)=0 .
$$

(ii) Then, we say that a function $f: I \rightarrow X$ is Stepanov- $[p, \phi, F]_{2}$-asymptotically $\omega$-periodic if and only if $\|f(\cdot+t+\omega)-f(\cdot+t)\| \in L^{p(\cdot)}[0,1]$ for any $t \in I$ and

$$
\lim _{|t| \rightarrow \infty} \phi\left(F(t)\|f(\cdot+t+\omega)-f(\cdot+t)\|_{L^{p(\cdot)}[0,1]}\right)=0 .
$$

Remark 1. The notion introduced in the above definitions is rather general. Let us only say the following: suppose that $I=\mathbb{R}$, the function $\phi(\cdot)$ is locally bounded, $\omega \in \mathbb{R}$ and $\sup _{t \in \mathbb{R}}\left[\|f(\cdot)\|_{L^{p(\cdot-\omega)}[t, t+1]}+\right.$ $\left.\|f(\cdot)\|_{L^{p(\cdot)}[t, t+1]}\right]<\infty$. Then, it readily follows that $f(\cdot)$ is Stepanov- $(p, \phi, F)$-asymptotically $\omega$-periodic for any function $F \in C_{0}(\mathbb{R}: X)$.

The notion introduced in the above definitions extends the notions of Stepanov $p$-quasi-asymptotical almost periodicity and the notion of Stepanov $p$-asymptotical $\omega$-periodicity $(1 \leq p<\infty)$. In case that $p(x) \equiv p \in[1, \infty)$, the Stepanov- $(p, \phi, F)$-classes coincide with the corresponding Stepanov- $[p, \phi, F]$-classes of functions. The most intriguing case, without any doubt, is that in which the functions $\mathrm{F}, F, \mathrm{~F}$ are identically equal to one and $\phi(x) \equiv x$; if this is the case and $p \in \mathcal{P}([0,1])$ (see Definitions 13-15), then we also say that the function $f: I \rightarrow X$ is Stepanov $p(x)$-quasi-asymptotically almost periodic (Stepanov $p(x)$-quasi-asymptotically uniformly recurrent, Stepanov $p(x)$-asymptotically $\omega$-periodic). In what follows, by $S^{p(x)} Q-A A P(I: X)$ $\left(S^{p(x)} Q-A U R(I: X), S^{p(x)} S A P_{\omega}(I: X)\right)$ we denote the collection of all such functions. It can be easily verified that the function $f: I \rightarrow X$ is Stepanov $p(x)$-quasi-asymptotically almost periodic (Stepanov $p(x)$-quasi-asymptotically uniformly recurrent, Stepanov $p(x)$-asymptotically $\omega$-periodic) if and only if the function $\hat{f}: I \rightarrow L^{p(x)}([0,1]: X)$ is quasi-asymptotically almost periodic (quasi-asymptotically uniformly recurrent, $S$-asymptotically $\omega$-periodic). This enables one to transfer the statements of Proposition 3 and Theorem 1 to the Stepanov classes (also see Reference [14], Theorem 2.10, Proposition 2.11), as well as to conclude that $S^{p(x)} S A P_{\omega}(I: X) \subseteq S^{p(x)} Q-A A P(I$ : $X) \subseteq S^{p(x)} Q-A U R(I: X)$ for any $p \in \mathcal{P}([0,1])$; see Reference [14] (Proposition 2.7).

Unfortunately, the spaces of (Stepanov $p(x)$-) quasi-asymptotically uniformly recurrent type functions are not closed under the operations of pointwise addition and multiplication. For instance, the consideration from Reference [14] (Example 2.16-Example 2.18) enables one to see that the following holds:

(i) There exist a continuous periodic function $f: \mathbb{R} \rightarrow \mathbb{R}$ and a function $g \in S A P_{2}(\mathbb{R}: \mathbb{R})$ such that the function $(f \cdot g)(\cdot)$ is not quasi-asymptotically uniformly recurrent.

(ii) There exist a Stepanov $p$-almost periodic function $f: \mathbb{R} \rightarrow \mathbb{R}$, where the exponent $p \geq 1$ can be chosen arbitrarily, and a function $g \in S A P_{4}(\mathbb{R}: \mathbb{R})$ such that $(f \cdot g)(\cdot)$ does not belong to the class $S^{1} Q-A U R(\mathbb{R}: \mathbb{R})$.

(iii) There exist a continuous periodic function $f:[0, \infty) \rightarrow \mathbb{R}$ and a function $g \in S A P_{4}([0, \infty): \mathbb{R})$ such that the function $(f+g)(\cdot)$ does not belong to the class $S^{1} Q-A U R([0, \infty): \mathbb{R})$.

We continue by stating the following 
Proposition 4. Suppose that $\phi(\cdot)$ is continuous for $t=0, \phi(0)=0$ and any of the functions $F, F, F$ is bounded. Then, any quasi-asymptotically uniformly recurrent function $f: I \rightarrow$ $X$ is Stepanov- $(p, \phi, F)$-quasi-asymptotically uniformly recurrent, Stepanov- $[p, \phi, F]$-quasi-asymptotically uniformly recurrent, as well as Stepanov- $(p, \phi, F)_{i}$-quasi-asymptotically uniformly recurrent and Stepanov- $[p, \phi, F]_{i}$-quasi-asymptotically uniformly recurrent $(i=1,2)$. The same statement holds for the corresponding classes of quasi-asymptotically almost periodic functions and S-asymptotically $\omega$-periodic functions.

Proof. We will provide the main details of the proof for the class of Stepanov- $[p, \phi, F]$-quasiasymptotically uniformly recurrent functions. Let $\left(\alpha_{n}\right)$ and $\left(M_{n}\right)$ be the sequences from Definition 9 , and let $\epsilon>0$. Then, there exists $\delta>0$ such that $|\phi(t)|=|\phi(t)-\phi(0)|<\epsilon,|t| \leq \delta$. Hence, $\sup \phi([0, \delta]) \leq \epsilon$. By our assumption, we have the existence of an integer $n_{0} \in \mathbb{N}$ such that

$$
\sup _{|t| \geq M_{n}}\left\|f\left(t+\alpha_{n}\right)-f(t)\right\| \leq \delta, \quad n \geq n_{0}
$$

Let $n \in \mathbb{N}$ with $n \geq n_{0}$ be fixed. Then, for every $t \geq M_{n}^{\prime}:=M_{n}+1$, we have $|t+x| \geq|t|-1 \geq M_{n}$, $x \in[0,1]$. This implies that, for every $t \geq M_{n}^{\prime}, x \in[0,1]$ and $\lambda \geq \epsilon$, we have $\phi\left(\| f\left(t+\alpha_{n}+x\right)-f(t+\right.$ $x) \|) / \lambda \leq 1, \varphi_{p(x)}\left(\phi\left(\left\|f\left(t+\alpha_{n}+x\right)-f(t+x)\right\|\right) / \lambda\right) \leq 1$ and therefore

$$
\int_{0}^{1} \varphi_{p(x)}\left(\phi\left(\left\|f\left(t+\alpha_{n}+x\right)-f(t+x)\right\|\right) / \lambda\right) d x \leq 1
$$

Thus,

$$
[\epsilon, \infty) \subseteq\left\{\lambda>0: \int_{0}^{1} \varphi_{p(x)}\left(\phi\left(\left\|f\left(t+\alpha_{n}+x\right)-f(t+x)\right\|\right) / \lambda\right) d x \leq 1\right\},
$$

which yields that

$$
\phi\left(\left\|f\left(\cdot+t+\alpha_{n}\right)-f(\cdot+t)\right\|\right)_{L^{p(\cdot)}[0,1]} \leq \epsilon, \quad n \geq n_{0} .
$$

This completes the proof by the boundedness of function $F(\cdot, \cdot)$.

As an immediate consequence, we have the following statement (also see Reference [10] (Proposition 4.5), where we proved that any almost periodic function $f: I \rightarrow X$ is $S^{p(x)}$-almost periodic for any $p \in \mathcal{P}([0,1]))$ :

Corollary 1. Let $\omega \in I$ and $p \in \mathcal{P}([0,1])$. Then, any quasi-asymptotically almost periodic (quasiasymptotically uniformly recurrent, S-asymptotically w-periodic) function $f: I \rightarrow X$ is Stepanov $p(x)$-quasi-asymptotically almost periodic (Stepanov $p(x)$-quasi-asymptotically uniformly recurrent, Stepanov $p(x)$-asymptotically $\omega$-periodic).

Using the trivial inequalities and Lemma 1, we can clarify a great number of inclusions for the introduced classes of functions. For instance, we can simply deduce the following (see Reference [10] (Proposition 4.3, Proposition 4.4, Proposition 4.8) for the first results in this direction, as well as Reference [12] (Remarks 2.6 and 2.10)):

(i) $S^{p(x)} S A P_{\omega}(I: X) \subseteq S^{1} S A P_{\omega}(I: X), S^{p(x)} Q-A A P(I: X) \subseteq S^{1} Q-A A P(I: X)$ and $S^{p(x)} Q-$ $A U R(I: X) \subseteq S^{1} Q-A U R(I: X)$.

(ii) Suppose $p \in D_{+}([0,1])$ and $1 \leq p^{-} \leq p(x) \leq p^{+}<\infty$ for a.e. $x \in[0,1]$. Then, we have $S^{p^{+}} S A P_{\omega}(I: X) \subseteq S^{p(x)} S A P_{\omega}(I: X) \subseteq S^{p^{-}} S A P_{\omega}(I: X), S^{p^{+}} Q-A A P(I: X) \subseteq S^{p(x)} Q-$ $A A P(I: X) \subseteq S^{p^{-}} Q-A A P(I: X)$, and $S^{p^{+}} Q-A U R(I: X) \subseteq S^{p(x)} Q-A U R(I: X) \subseteq$ $S^{P^{-}} Q-A U R(I: X)$. 
(iii) Suppose $p, q \in \mathcal{P}([0,1])$ and $p \leq q$ a.e. on $[0,1]$. Then, we have $S^{q(x)} S A P_{\omega}(I: X) \subseteq$ $S^{p(x)} S A P_{\omega}(I: X), S^{q(x)} Q-A A P(I: X) \subseteq S^{p(x)} Q-A A P(I: X)$ and $S^{q(x)} Q-A U R(I: X) \subseteq$ $S^{p(x)} Q-A U R(I: X)$.

These inclusions can be simply transferred and reformulated for the general classes of functions introduced in Definitions 10-12 and Definitions 13-15; details can be left to the interested readers.

The first part of subsequent result is very similar to Reference [12] (Proposition 2.5); the proof is based on the use of Jensen integral inequality and therefore omitted.

Proposition 5. (i) Suppose that $\phi(\cdot)$ is convex, $p(x) \equiv 1$ and $f \in L_{l o c}^{1}(I: X)$. If $f(\cdot)$ is Stepanov $(p, 1, F)$-quasi asymptotically uniformly recurrent, then $f(\cdot)$ is Stepanov $(p, 1, F)_{1}$-quasi asymptotically uniformly recurrent. If the function $\phi(\cdot)$ is concave, then the above inclusion reverses.

(ii) Suppose that there exists a function $\varphi:[0, \infty) \rightarrow[0, \infty)$ such that $\phi(x y) \leq \varphi(x) \phi(y)$ for all $x, y \geq 0$. If $f(\cdot)$ is Stepanov $(p, \phi, F)_{1}$-quasi asymptotically uniformly recurrent, resp. Stepanov $[p, \phi, F]_{1}$-quasi asymptotically uniformly recurrent, then $f(\cdot)$ is Stepanov $\left(p, \phi, F_{1}\right)_{2}$-quasi asymptotically uniformly recurrent, resp. Stepanov $\left[p, \phi, F_{1}\right]_{2}$-quasi asymptotically uniformly recurrent, provided that $F=\varphi \circ F_{1}$.

Furthermore, the same statements hold for the corresponding classes of quasi-asymptotically almost periodic functions and S-asymptotically w-periodic functions.

The basic structural properties of quasi-asymptotically almost periodic functions clarified in Reference [14] (Theorem 2.13) can be formulated in our framework, for the general classes of functions introduced in this subsection, as well. We leave readers to make this explicit.

If $p \in[1, \infty)$, then any Stepanov $p$-quasi-asymptotically almost periodic function is Weyl $p$-almost periodic (see Reference [14], Proposition 2.12). The argumentation used in the proof of this result also shows that any Stepanov $p$-quasi-asymptotically uniformly recurrent function is Weyl-p-uniformly recurrent. In general case, we will state and prove only one result regarding the notion introduced in Definitions 3 and 10:

Proposition 6. Suppose that the function $f: I \rightarrow X$ is Stepanov- $(p, \phi, F)$-quasi-asymptotically uniformly recurrent. If $F_{1}:(0, \infty) \times I \rightarrow(0, \infty)$ satisfies

$$
\lim _{n \rightarrow+\infty} \limsup _{l \rightarrow+\infty} \sup _{t \in I} F_{1}(l, t)\left[\frac{1}{F(t, n)}+\cdots+\frac{1}{F(\lfloor t+l\rfloor, n)}\right]<\infty
$$

and

$$
\lim _{l \rightarrow+\infty} \sup _{t \in I} F_{1}(l, t)=0,
$$

then the function $f(\cdot)$ is Weyl- $\left(p(x), \phi, F_{1}\right)$-uniformly recurrent.

Proof. By our assumption, we have $\phi(\|f(\cdot+\tau)-f(\cdot)\|) \in L^{p(\cdot)}(K)$ for any $\tau \in I$ and any compact set $K \subseteq I$; furthermore, we know that there exists a strictly increasing sequence $\left(\alpha_{n}\right)$ of positive real numbers tending to plus infinity and a sequence $\left(M_{n}\right)$ of positive real numbers such that (6) holds. We will prove that (4) holds with the function $F(\cdot, \cdot)$ replaced therein with the function $F_{1}(\cdot, \cdot)$. Let $n \in \mathbb{N}$ and $l>0$ be fixed. If $t \in I$, then there exist four possibilities:

1. $|t| \geq M_{n}$ and $|t+l| \geq M_{n}$

2. $|t| \geq M_{n}$ and $|t+l| \leq M_{n}$;

3. $|t| \leq M_{n}$ and $|t+l| \geq M_{n}$;

4. $|t| \leq M_{n}$ and $|t+l| \leq M_{n}$.

The consideration is similar for all these cases, and we will give the proof for case (1.), only. If $t \geq 0$, then we have $t \geq M_{n}, t+l \geq M_{n}$ and therefore 


$$
\begin{aligned}
& {\left[F_{1}(l, t)\left[\phi\left(\left\|f\left(\cdot+\alpha_{n}\right)-f(\cdot)\right\|\right)_{L^{p(\cdot)}[t, t+l]}\right]\right]} \\
& \leq F_{1}(l, t)\left[\frac{\epsilon}{F(t, n)}+\cdots+\frac{\epsilon}{F(\lfloor t+l\rfloor, n)}\right]
\end{aligned}
$$

also see (3). Employing condition (7), we immediately get (4). If $t \leq 0$, then we have $t \leq-M_{n}$ and $t+l \geq M_{n}$ for a sufficiently large $l>0$ (it suffices to consider only such case because, in (4), we operate with $\left.\lim \sup _{l \rightarrow+\infty} \cdot\right)$. We have

$$
\begin{aligned}
& {\left[F_{1}(l, t)\left[\phi\left(\left\|f\left(\cdot+\alpha_{n}\right)-f(\cdot)\right\|\right)_{L^{p(\cdot)}[t, t+l]}\right]\right]} \\
& \leq\left[F _ { 1 } ( l , t ) \left[\phi\left(\left\|f\left(\cdot+\alpha_{n}\right)-f(\cdot)\right\|\right)_{L^{p(\cdot)}\left[t,-M_{n}\right]}+\phi\left(\left\|f\left(\cdot+\alpha_{n}\right)-f(\cdot)\right\|\right)_{L^{p(\cdot)}\left[-M_{n}, M_{n}\right]}\right.\right. \\
& \left.\left.+\phi\left(\left\|f\left(\cdot+\alpha_{n}\right)-f(\cdot)\right\|\right)_{L^{p(\cdot)}\left[M_{n}, t+l\right]}\right]\right] \\
& \leq F_{1}(l, t)\left[\left(\frac{\epsilon}{F(t, n)}+\cdots+\frac{\epsilon}{F\left(t+\left\lfloor-t-M_{n}\right\rfloor, n\right)}\right)\right. \\
& \left.+\left(\frac{\epsilon}{F\left(M_{n}, n\right)}+\cdots+\frac{\epsilon}{F\left(M_{n}+\left\lfloor t+l-M_{n}\right\rfloor, n\right)}\right)\right] \\
& +F_{1}(l, t) \phi\left(\left\|f\left(\cdot+\alpha_{n}\right)-f(\cdot)\right\|\right)_{L^{p(\cdot)}\left[-M_{n}, M_{n}\right]} \\
& \leq 2 F_{1}(l, t)\left[\frac{\epsilon}{F(t, n)}+\cdots+\frac{\epsilon}{F(\lfloor t+l\rfloor, n)}\right] \\
& +F_{1}(l, t) \phi\left(\left\|f\left(\cdot+\alpha_{n}\right)-f(\cdot)\right\|\right)_{L^{p(\cdot)}\left[-M_{n}, M_{n}\right]}
\end{aligned}
$$

Using (7) and (8), we get (4).

\subsection{Composition Principles for the Class of Quasi-Asymptotically Uniformly Recurrent Functions}

In this subsection, we will briefly consider quasi-asymptotically uniformly recurrent functions depending on two parameters and related composition theorems (for the sake of brevity, we will say only a few words about the Stepanov classes). In order to unify several different approaches used in the existing literature, in this subsection, we will assume that $\mathbf{B} \subseteq P(Y)$, where $P(Y)$ denotes the power set of $Y$; usually, B denotes the collection of all bounded subsets of $Y$ or all compact subsets of $Y$.

Definition 16. (i) By $C_{0, \mathbf{B}}(I \times Y: X)$, we denote the space of all continuous functions $H: I \times Y \rightarrow X$ such that $\lim _{|t| \rightarrow+\infty} H(t, y)=0$ uniformly for $y$ in any subset $B \in \mathbf{B}$.

(ii) A continuous function $F: I \times Y \rightarrow X$ is said to be uniformly continuous on $\mathbf{B}$, uniformly for $t \in I$ if and only if for every $\epsilon>0$ and for every $B \in \mathbf{B}$ there exists a number $\delta_{\epsilon, B}>0$ such that $\|F(t, x)-F(t, y)\| \leq$ $\epsilon$ for all $t \in I$ and all $x, y \in B$ satisfying that $\|x-y\| \leq \delta_{\epsilon, B}$.

We continue by introducing the following definition:

Definition 17. Suppose that $F: I \times Y \rightarrow X$ is a continuous function and $\mathbf{B} \subseteq P(Y)$. Then, we say that $F(\cdot, \cdot)$ is quasi-asymptotically uniformly recurrent, uniformly on $\mathbf{B}$ if and only if for every $B \in \mathbf{B}$ there exists a strictly 
increasing sequence $\left(\alpha_{n}\right)$ of positive real numbers tending to plus infinity and a sequence $\left(M_{n}\right)$ of positive real numbers such that:

$$
\lim _{n \rightarrow+\infty} \sup _{|t| \geq M_{n}}\left\|F\left(t+\alpha_{n}, x\right)-F(t, x)\right\|=0, \quad x \in B
$$

Denote by $Q-A U R_{\mathbf{B}}(I \times Y: X)$ the set consisting of all quasi-asymptotically unifomly recurrent, uniformly on $\mathbf{B}$ functions from $I \times Y$ into $X$.

Using the argumentation employed in the proofs of Reference [2] (Theorems 3.30 and 3.31), we may deduce the following results:

Theorem 2. Suppose that $\mathbf{B} \subseteq P(Y), R(f) \in \mathbf{B}, F \in Q-A U R_{\mathbf{B}}(I \times Y: X)$ and $f \in Q-A U R(I: Y)$. If there exist a finite number $L>0$ such that

$$
\|F(t, x)-F(t, y)\|_{Y} \leq L\|x-y\|, x, y \in Y, t \in I
$$

a strictly increasing sequence $\left(\alpha_{n}\right)$ of positive real numbers tending to plus infinity and a sequence $\left(M_{n}\right)$ of positive real numbers such that (9) holds with $B=R(f)$ and (2) holds, then the function $t \mapsto F(t, f(t)), t \in I$ belongs to the class $Q-A U R(I: X)$.

Theorem 3. Suppose that $\mathbf{B} \subseteq P(Y), R(f) \in \mathbf{B}, F \in Q-A U R_{\mathbf{B}}(I \times Y: X)$ and $f \in Q-A U R(I: Y)$. If $F: I \times Y \rightarrow X$ is uniformly continuous on $\mathbf{B}$, uniformly for $t \in I$ and there exist a strictly increasing sequence $\left(\alpha_{n}\right)$ of positive real numbers tending to plus infinity and a sequence $\left(M_{n}\right)$ of positive real numbers such that (9) holds with $B=R(f)$ and (2) holds, then the function $t \mapsto F(t, f(t)), t \in I$ belongs to the class $Q-\operatorname{AUR}(I: X)$.

Similarly as in Definition 17, we can introduce the notion of a quasi-asymptotically almost periodic, uniformly on B function and the notion of a $S$-asymptotically $\omega$-periodic, uniformly on B function. Theorems 2 and 3 continue to hold in this framework.

In Reference [14] (Definition 2.22), we introduced the notion of a Stepanov $p$-quasi-asymptotically almost periodic function depending on two parameters $(1 \leq p<\infty)$; the notion of a Stepanov $p(x)$-quasi-asymptotically almost periodic function (Stepanov $p(x)$-quasi-asymptotically uniformly recurrent function, Stepanov $p(x)$-asymptotically $\omega$-periodic function) can be introduced in a similar fashion. The interested reader may try to extend Reference [14] (Theorems 2.23 and 2.24) in this context (also see Reference [24]).

\subsection{Invariance of Generalized Quasi-Asymptotical Uniform Recurrence under the Actions of} Convolution Products

This subsection investigates the invariance of generalized quasi-asymptotical uniform recurrence under the actions of finite and infinite convolution products. Using the same arguments as in the proofs of Reference [14] (Propositions 3.1 and 3.2), we can deduce the validity of the following statement:

Proposition 7. ( $i)$ Suppose that $(R(t))_{t>0} \subseteq L(X, Y)$ is a strongly continuous operator family and $\int_{0}^{\infty}\|R(s)\| d s<\infty$. If $f \in Q-A U R([0, \infty): X) \cap L^{\infty}([0, \infty): X)$, then the function $F(\cdot)$, defined by

$$
\mathrm{F}(t):=\int_{0}^{t} R(t-s) f(s) d s, t \geq 0,
$$

belongs to the class $Q-A U R([0, \infty): Y) \cap L^{\infty}([0, \infty): Y)$. 
(ii) Suppose that $(R(t))_{t>0} \subseteq L(X, Y)$ is a strongly continuous operator family and $\int_{0}^{\infty}\|R(s)\| d s<\infty$. If $f \in Q-A U R(\mathbb{R}: X) \cap L^{\infty}(\mathbb{R}: X)$, then the function $\mathbf{F}(t)$, defined by

$$
\mathbf{F}(t):=\int_{-\infty}^{t} R(t-s) f(s) d s, \quad t \in \mathbb{R},
$$

belongs to the class $Q-A U R(\mathbb{R}: Y) \cap L^{\infty}(\mathbb{R}: Y)$.

Concerning the invariance of Stepanov quasi-asymptotically almost periodic properties analyzed in the previous subsection, it would be really difficult and rather long to examine all introduced classes. Primarily from this reason, we will focus our attention on the notion introduced in Definition 13, only.

The following result admits a simple reformulation for the corresponding classes of quasi-asymptotically almost periodic functions and $S$-asymptotically $\omega$-periodic functions:

Proposition 8. Suppose that $\left(a_{k}\right)$ is a sequence of positive real numbers such that $\sum_{k=0}^{\infty} a_{k}=1, \varphi:[0, \infty) \rightarrow$ $[0, \infty), \phi:[0, \infty) \rightarrow[0, \infty)$ is a convex monotonically increasing function satisfying $\phi(x y) \leq \varphi(x) \phi(y)$ for all $x, y \geq 0, p, q \in \mathcal{P}([0,1]), 1 / p(x)+1 / q(x)=1$ and $(R(t))_{t>0} \subseteq L(X, Y)$ is a strongly continuous operator family satisfying that

$$
M:=\sum_{k=0}^{\infty} a_{k} \varphi\left(a_{k}^{-1}\right)[\varphi(\|R(\cdot+k)\|)]_{L^{q(\cdot)}[0,1]}<\infty .
$$

Suppose, further, that, for every $x \in \mathbb{R}$, we have $\int_{-x}^{\infty}\|R(v+x)\|\|\check{f}(v)\| d v<\infty_{\text {, }}$ as well as that $\check{f}(\cdot)$ is Stepanov-[p, $\phi, F]$-quasi-asymptotically uniformly recurrent, $M_{1}:=\sup _{t \in \mathbb{R}}[\phi(\|f(t-s)\|)]_{L^{p(s)}[0,1]}<\infty$, $F_{1}:(0, \infty) \times \mathbb{N} \rightarrow(0, \infty)$ is bounded and satisfies that there exists a finite real constant $c>0$ such that $F_{1}(t, n) \leq c F(t, n)$ for all $t>0$ and $n \in \mathbb{N}$. Then, the function $\mathbf{F}: \mathbb{R} \rightarrow Y$, given by (12), is well-defined and Stepanov- $\left[\infty, \phi, F_{1}\right]$-quasi-asymptotically uniformly recurrent.

Proof. Since, for every $x \in \mathbb{R}$, we have $\int_{-x}^{\infty}\|R(v+x)\|\|\breve{f}(v)\| d v<\infty$, it can be easily verified that the function $\mathbf{F}(\cdot)$ is well defined, as well as that the integral which defines $\mathbf{F}(x+\tau)-\mathbf{F}(x)$ is absolutely convergent for every $x \in \mathbb{R}$ and $\tau \in \mathbb{R}$ (also see the proof of Reference [12], Theorem 4.1). For the rest, let $\left(\alpha_{n}\right)$ and $\left(M_{n}\right)$ be the sequences from Definition 13, for the function $f(\cdot)$ replaced therein with the function $\check{f}(\cdot)$. Let $\epsilon>0$ be given, and let $n_{0} \in \mathbb{N}$ be such that $\phi\left(\left\|f\left(t+\alpha_{n}+\cdot\right)-f(t+\cdot)\right\|\right)_{L^{p(\cdot)}[0,1]}<$ $\epsilon / F(t, n), n \geq n_{0},|t| \geq M_{n}$. Clearly, there exists $k_{0}(\epsilon) \in \mathbb{N}$ such that

$$
\sum_{k=k_{0}(\epsilon)}^{\infty} a_{k} \varphi\left(a_{k}^{-1}\right)[\varphi(\|R(\cdot+k)\|)]_{L^{q(\cdot)}[0,1]}<\epsilon
$$

Let $\alpha_{n} \geq k_{0}(\epsilon)$ for $n \geq n_{1}$. Let $n \in \mathbb{N}$ with $n \geq \max \left(n_{0}, n_{1}\right)$ be fixed, and let $|t| \geq M_{n}^{\prime}:=M_{n}+\alpha_{n}+2$. Then, for each $x \in[0,1]$, we have (apply the Jensen inequality, (1) and the Hölder inequality)

$$
\begin{aligned}
& \left\|\mathbf{F}\left(t+x+\alpha_{n}\right)-\mathbf{F}(t+x)\right\| \\
& \leq \phi\left(\int_{0}^{\infty}\|R(s)\|\left\|f\left(x+t+\alpha_{n}-s\right)-f(x+t-s)\right\| d s\right) \\
& =\phi\left(\sum_{k=0}^{\infty} a_{k} \int_{0}^{1} a_{k}^{-1}\|R(s+k)\|\left\|f\left(x+t+\alpha_{n}-k-s\right)-f(x+t-k-s)\right\| d s\right) \\
& \leq \sum_{k=0}^{\infty} a_{k} \phi\left(\int_{0}^{1} a_{k}^{-1}\|R(s+k)\|\left\|f\left(x+t+\alpha_{n}-k-s\right)-f(x+t-k-s)\right\| d s\right)
\end{aligned}
$$




$$
\begin{aligned}
& \leq \sum_{k=0}^{\infty} a_{k} \varphi\left(a_{k}^{-1}\right) \int_{0}^{1} \phi\left(\|R(s+k)\|\left\|f\left(x+t+\alpha_{n}-k-s\right)-f(x+t-k-s)\right\|\right) d s \\
& \leq 2 \sum_{k=0}^{\infty} a_{k} \varphi\left(a_{k}^{-1}\right)[\varphi(\|R(\cdot+k)\|)]_{L^{q(\cdot)}[0,1]} \\
& \times\left[\phi\left(\left\|f\left(x+t+\alpha_{n}-k-\cdot\right)-f(x+t-k-\cdot)\right\|\right)\right]_{L^{p(\cdot)}[0,1]^{\prime}}
\end{aligned}
$$

which implies that, for $t \leq-M_{n}^{\prime}$, we have

$$
\begin{aligned}
\left\|\mathbf{F}\left(t+x+\alpha_{n}\right)-\mathbf{F}(t+x)\right\| & \leq 2 \sum_{k=0}^{\infty} a_{k} \varphi\left(a_{k}^{-1}\right)[\varphi(\|R(\cdot+k)\|)]_{L^{q(\cdot)}[0,1]} \frac{\epsilon}{F(t, n)} \\
& \leq 2 c \sum_{k=0}^{\infty} a_{k} \varphi\left(a_{k}^{-1}\right)[\varphi(\|R(\cdot+k)\|)]_{L^{q(\cdot)}[0,1]} \frac{\epsilon}{F_{1}(t, n)} .
\end{aligned}
$$

If $t \geq M_{n}^{\prime}$, then we have $\left\lfloor t-M_{n}\right\rfloor \geq k_{0}(\epsilon)$, and (14) implies

$$
\begin{aligned}
& \left\|\mathbf{F}\left(t+x+\alpha_{n}\right)-\mathbf{F}(t+x)\right\| \\
& \quad \leq 2 \sum_{k=0}^{\left\lfloor t-M_{n}\right\rfloor} a_{k} \varphi\left(a_{k}^{-1}\right)[\varphi(\|R(\cdot+k)\|)]_{L^{q(\cdot)}[0,1]} \\
& \quad \times\left[\phi\left(\left\|f\left(x+t+\alpha_{n}-k-s\right)-f(x+t-k-s)\right\|\right)\right]_{L^{p(s)}[0,1]} \\
& +2 \sum_{k=\left\lfloor t-M_{n}\right\rfloor}^{\left\lceil t+M_{n}\right\rceil} a_{k} \varphi\left(a_{k}^{-1}\right)[\varphi(\|R(\cdot+k)\|)]_{L^{q(\cdot)}[0,1]} \\
& \quad \times\left[\phi\left(\left\|f\left(x+t+\alpha_{n}-k-s\right)-f(x+t-k-s)\right\|\right)\right]_{L^{p(s)}[0,1]} \\
& +2 \sum_{k=\left\lceil t+M_{n}\right\rceil}^{\infty} a_{k} \varphi\left(a_{k}^{-1}\right)[\varphi(\|R(\cdot+k)\|)]_{L^{q(\cdot)}[0,1]} \\
& \quad \times\left[\phi\left(\left\|f\left(x+t+\alpha_{n}-k-s\right)-f(x+t-k-s)\right\|\right)\right]_{L^{p(s)}[0,1]} \\
& \quad \leq 2 \frac{\epsilon}{F(t, n)}\left(\sum_{k=0}^{\left\lfloor t-M_{n}\right\rfloor}+\sum_{k=\left\lceil t+M_{n}\right]}^{\infty}\right) a_{k} \varphi\left(a_{k}^{-1}\right)[\varphi(\|R(\cdot+k)\|)]_{L^{q(\cdot)}[0,1]}+\epsilon \cdot \varphi(2) \cdot M_{1} \\
& \quad \leq 2 c \frac{\epsilon}{F_{1}(t, n)}\left(\sum_{k=0}^{\left\lfloor t-M_{n}\right\rfloor}+\sum_{k=\left\lceil t+M_{n}\right\rceil}^{\infty}\right) a_{k} \varphi\left(a_{k}^{-1}\right)[\varphi(\|R(\cdot+k)\|)]_{L^{q(\cdot)}[0,1]}+\epsilon \cdot \varphi(2) \cdot M_{1},
\end{aligned}
$$

since

$$
\begin{aligned}
& {\left[\phi\left(\left\|f\left(x+t+\alpha_{n}-k-s\right)-f(x+t-k-s)\right\|\right)\right]_{L^{p(s)}[0,1]}} \\
& \leq\left[\phi\left(\left\|f\left(x+t+\alpha_{n}-k-s\right)\right\|+\|f(x+t-k-s)\|\right)\right]_{L^{p(s)}[0,1]} \\
& \leq \varphi(2)\left[\frac{1}{2} \phi\left(\left\|f\left(x+t+\alpha_{n}-k-s\right)\right\|\right)+\frac{1}{2} \phi(\|f(x+t-k-s)\|)\right]_{L^{p(s)}[0,1]} \leq \varphi(2) \cdot M_{1} .
\end{aligned}
$$

This simply completes the proof.

We will also state the following special corollary, which generalizes Reference [14] (Proposition 3.4): 
Proposition 9. Suppose that $q \in \mathcal{P}([0,1]), 1 / p(x)+1 / q(x)=1$ and $(R(t))_{t>0} \subseteq L(X, Y)$ is a strongly continuous operator family satisfying that $M:=\sum_{k=0}^{\infty}\|R(\cdot+k)\|_{L^{q(x)}[0,1]}<\infty$. If $\check{f}: \mathbb{R} \rightarrow X$ is Stepanov $p(x)$-quasi-asymptotically uniformly recurrent (Stepanov $p(x)$-quasi-asymptotically almost periodic, Stepanov $p(x)$-asymptotically $\omega$-periodic) and $S^{p(x)}$-bounded, then the function $\mathbf{F}: \mathbb{R} \rightarrow Y$, given by (12), is well defined, bounded and quasi-asymptotically uniformly recurrent (quasi-asymptotically almost periodic, S-asymptotically $\omega$-periodic).

Proof. We will consider the Stepanov $p(x)$-quasi-asymptotically uniformly recurrent functions, only. Since $\sum_{k=0}^{\infty}\|R(\cdot+k)\|_{L^{q(x)}[0,1]}<\infty$ and $\breve{f}(\cdot)$ is $S^{p(x)}$-bounded, we can apply the same arguments as in the proofs of Reference [10] (Proposition 6.1) and Reference [11] (Proposition 5.1) in order to see that the function $\mathbf{F}(\cdot)$ is bounded and continuous. The remainder of proof follows from the computations carried out in the proof of Proposition 10, with $p(x)=\varphi(x)=\phi(x)=x$ and $F(t, n)=F_{1}(t, n)=1$.

The following result regarding the finite convolution product admits a reformulation for the corresponding classes of quasi-asymptotically almost periodic functions and S-asymptotically $\omega$-periodic functions, as well:

Proposition 10. Suppose that $\left(a_{k}\right)$ is a sequence of positive real numbers such that $\sum_{k=0}^{\infty} a_{k}=1, \varphi:[0, \infty) \rightarrow$ $[0, \infty), \phi:[0, \infty) \rightarrow[0, \infty)$ is a convex monotonically increasing function satisfying $\phi(x y) \leq \varphi(x) \phi(y)$ for all $x, y \geq 0, p, q \in \mathcal{P}([0,1]), 1 / p(x)+1 / q(x)=1$ and $(R(t))_{t>0} \subseteq L(X, Y)$ is a strongly continuous operator family satisfying that (13) holds. Suppose, further, that the mapping $\mathrm{F}:[0, \infty) \rightarrow Y$, given by (11), is well-defined, as well as that $f(\cdot)$ is Stepanov- $[p, \phi, F]$-quasi-asymptotically uniformly recurrent,

$$
M_{1}:=\sup _{t \geq 0} \sup _{t \in[0, s]}[\phi(\|f(t-s)\|)]_{L^{p(s)}[0,1]}<\infty,
$$

$F_{1}:(0, \infty) \times \mathbb{N} \rightarrow(0, \infty)$ is bounded and satisfies that there exists a finite real constant $c>0$ such that $F_{1}(t, n) \leq c F(t, n)$ for all $t>0$ and $n \in \mathbb{N}$. Then, the function $\mathrm{F}(\cdot)$ is Stepanov- $\left[\infty, \phi, F_{1}\right]$-quasi-asymptotically uniformly recurrent.

Proof. The proof is very similar to the proof of Proposition 10, and we will only outline two details. Let $\epsilon>0$ be fixed, and let the numbers $M_{n}>0$ and $k_{0}(\epsilon), n_{0}, n_{1} \in \mathbb{N}$ be as above. Then, for each $x \in[0,1],|t| \geq M_{n}^{\prime}+\alpha_{n}+2$ and $n \in \mathbb{N}$ with $n \geq \max \left(n_{0}, n_{1}\right)$, we have

$$
\begin{aligned}
\phi( & \left.\left\|F\left(x+t+k+\alpha_{n}\right)-F(x+t+k)\right\|\right) \\
& \leq \sum_{k=0}^{[t\rceil} a_{k} \varphi\left(a_{k}^{-1}\right)[\varphi(\|R(\cdot+k)\|)]_{L^{q(\cdot)}[0,1]} \\
& \times\left[\phi\left(\left\|f\left(x+t+k+\alpha_{n}-s\right)-f(x+t+k-s)\right\|\right)\right]_{L^{p(s)}[0,1]}
\end{aligned}
$$

After that, we can decompose the sum $\sum_{k=0}^{[t]}$. into two parts:

$$
\sum_{k=0}^{\lceil t\rceil} \cdot=\sum_{k=0}^{k_{0}(\epsilon)} \cdot+\sum_{k=k_{0}(\epsilon)}^{\lceil t\rceil},
$$

and apply the similar arguments. This completes the proof in a routine manner.

Similarly, we can deduce the following extension of Reference [14] (Proposition 3.3) (also see the proof of Reference [11], Proposition 5.1): 
Proposition 11. Suppose that $q \in \mathcal{P}([0,1]), 1 / p(x)+1 / q(x)=1$ and $(R(t))_{t>0} \subseteq L(X, Y)$ is a strongly continuous operator family satisfying that $M:=\sum_{k=0}^{\infty}\|R(\cdot+k)\|_{L^{q(x)}[0,1]}<\infty$. If $f:[0, \infty) \rightarrow X$ is Stepanov $p(x)$-quasi-asymptotically almost periodic (Stepanov $p(x)$-quasi-asymptotically uniformly recurrent, Stepanov $p(x)$-asymptotically $\omega$-periodic), $f(t-\cdot) \in L^{p(x)}[0, t]$ for $0<t \leq 1$ and

$$
\sup _{k \in \mathbb{N}_{0}} \sup _{t \geq 0}\|f(t+k-\cdot)\|_{L^{p(\cdot)}[0,1]}<\infty,
$$

then the function $\mathrm{F}:[0, \infty) \rightarrow Y$, given by (11), is well-defined, bounded and quasi-asymptotically almost periodic (quasi-asymptotically uniformly recurrent, S-asymptotically $\omega$-periodic).

Remark 2. We would like to note that it is very difficult to remove the assumption on the boundedness of function $f(\cdot)$ in Proposition 7, resp. the Stepanov $p(x)$-boundedness of functions in Propositions 9-11, in contrast to our recent research study [21].

\section{Applications to the Abstract Volterra Integro-Differential Equations}

Concerning possible applications of our theoretical results to the abstract Volterra integrodifferential equations in Banach spaces, we would like to say first a few words about the abstract nonautonomous differential equations of first order. In the first part of Reference [14] (Section 4), we investigated the generalized almost periodic properties of the mild solutions to the abstract Cauchy problems

$$
\begin{gathered}
u^{\prime}(t)=A(t) u(t)+f(t), \quad t \in \mathbb{R}, \\
u^{\prime}(t)=A(t) u(t)+f(t), \quad t>0 ; u(0)=x,
\end{gathered}
$$

where the operator family $A(\cdot)$ satisfies certain conditions. In Reference [14] (Section 4.1), we investigated the generalized almost periodic properties of the semilinear analogues to the abstract Cauchy problems (15) and (16).

The statement of Reference [14] (Theorem 4.1) can be straightforwardly extended for the inhomogeneities $f \in S^{p(x)} Q-A A P([0, \infty): X)$ by replacing the number $q$ in the equation [14] (4.1) with the function $q(x)$ and using the translation $\cdot \mapsto \cdot+k(1 / p(x)+1 / q(x)=1)$ therein; we can also consider the inhomogeneities $f \in S^{p(x)} Q-A U R([0, \infty): X)$ which are Stepanov $p(x)$-bounded, by slightly modifying the equation [14] (4.1) in the formulation of this result. Similar comments can be made for Reference [14] (Theorem 4.3). Concerning semilinear problems, the statements of Reference [14] (Theorem 4.6, Theorem 4.7) can be reformulated by replacing the space $Q-A A P(I: X)$ with the space $B Q-A U R_{\left(\alpha_{n}\right)}(I: X)$ consisting of all bounded functions $f: I \rightarrow X$ which are quasi-asymptotically uniformly recurrent and for which there exists a fixed sequence $\left(\alpha_{n}\right)$ of positive real numbers such that (5) holds; equipped with the metric $d(\cdot, \cdot):=\|\cdot-\cdot\|_{\infty}$, this space becomes a complete metric space; the conclusions established in Reference [14] (Example 2.8) can be reexamined in this context, as well.

Concerning the multivalued linear approach to the abstract degenerate Volterra integro-differential equations in Banach spaces, we refer the reader to the research monographs [25] by Favini, Yagi, and [26] by Kostić. In what follows, we assume that a multivalued linear operator $\mathcal{A}$ in $X$ satisfies condition [5] ((P)). Let $\gamma \in(0,1)$, and let the operator families $\left(S_{\gamma}(t)\right)_{t>0}$ and $\left(R_{\gamma}(t)\right)_{t>0}$ possess the same meaning as in Reference [5]. Then, we know that

$$
\left\|S_{\gamma}(t)\right\|=O\left(t^{-\gamma}\right), t \geq 1 \text { and }\left\|R_{\gamma}(t)\right\|=O\left(t^{\gamma-1}+t^{-\gamma-1}\right), \quad t>0
$$


We assume that $x_{0} \in X$ belongs to the continuity set of $\left(S_{\gamma}(t)\right)_{t>0}$, i.e., that $\lim _{t \rightarrow 0+} S_{\gamma}(t) x_{0}=x_{0}$. Due to the first estimate in (17), we have that the mapping $t \mapsto S_{\gamma}(t) x_{0}, t \geq 0$ belongs to the space $C_{0}([0, \infty): X)$.

We will use the following notion of the Caputo fractional derivatives of order $\gamma$ (for more details about fractional calculus and fractional differential equations, we refer the reader to the monograph [27] by Kilbas, Srivastava, and Trujillo, and the references cited in Reference [13]). The Caputo fractional derivative of order $\gamma$, denoted by $\mathbf{D}_{t}^{\gamma} u(t)$, is defined for those functions $u:[0, T] \rightarrow X$ for which $u_{\mid(0, T]}(\cdot) \in C((0, T]: E), u(\cdot)-u(0) \in L^{1}((0, T): X)$ and $g_{1-\gamma} \times(u(\cdot)-u(0)) \in W^{1,1}((0, T): X)$, by

$$
\mathbf{D}_{t}^{\gamma} u(t):=\frac{d}{d t}\left[g_{1-\gamma} \times(u(\cdot)-u(0))\right](t), \quad t \in(0, T] ;
$$

here, $W^{1,1}((0, T): X)$ denotes the usual Sobolev space of order 1 . By a mild solution of the abstract semilinear Cauchy inclusion

$$
(D F P)_{F, \gamma, s}:\left\{\begin{array}{c}
\mathbf{D}_{t}^{\gamma} u(t) \in \mathcal{A} u(t)+F(t, u(t)), t>0, \\
u(0)=x_{0},
\end{array}\right.
$$

we mean any function $u \in C([0, \infty): X)$ satisfying that

$$
u(t)=S_{\gamma}(t) x_{0}+\int_{0}^{t} R_{\gamma}(t-s) F(s, u(s)) d s, \quad t \geq 0 .
$$

Now, we are in a position to state the following result:

Theorem 4. Suppose that the function $F: \mathbb{R} \times X \rightarrow X$ is continuous and satisfies that, for each bounded subset $B$ of $X$, there exist a finite real constant $M_{B}>0$ and a sequence $\left(M_{n}\right)$ of positive real numbers such that (9) holds and $\sup _{t \in \mathbb{R}} \sup _{x \in B}\|F(t, x)\| \leq M_{B}$. Let there exist a finite number $L>0$ such that (10) holds, and let there exist an integer $n \in \mathbb{N}$ such that $A_{n}<1$, where

$$
\begin{aligned}
A_{n}:= & \sup _{t \geq 0} \int_{0}^{t} \int_{0}^{x_{n}} \cdots \int_{0}^{x_{2}} L^{n}\left\|R_{\gamma}\left(t-x_{n}\right)\right\| \\
& \times \prod_{i=2}^{n}\left\|R_{\gamma}\left(x_{i}-x_{i-1}\right)\right\| d x_{1} d x_{2} \cdots d x_{n} .
\end{aligned}
$$

Then, the abstract fractional Cauchy inclusion $(D F P)_{F, \gamma, s}$ has a unique solution which belongs to the space $B Q-A U R_{\left(\alpha_{n}\right)}([0, \infty): X)$.

Proof. Set, for every $u \in C_{b}([0, \infty): X)$,

$$
(\mathrm{Y} u)(t):=S_{\gamma}(t) x_{0}+\int_{0}^{t} R_{\gamma}(t-s) F(s, u(s)) d s, \quad t \geq 0 .
$$

Suppose that $u \in B Q-A U R_{\left(\alpha_{n}\right)}([0, \infty): X)$. Then, $R(u)=B$ is a bounded set and our assumption implies that the mapping $t \mapsto F(t, u(t)), t \in \mathbb{R}$ is bounded. Applying Theorem 2, we have that the function $F(\cdot, u(\cdot))$ is quasi-asymptotically uniformly recurrent. After that, we can employ Proposition 7 (i) and Proposition 2 (there is no need to say that we can retain the same sequence $\left(\alpha_{n}\right)$ after applying the above-mentioned statements, with the meaning clear) in order to see that $Y u \in B Q-A U R_{\left(\alpha_{n}\right)}([0, \infty)$ : $X)$. Hence, the mapping $Y(\cdot)$ is well defined. Since

$$
\left\|\left(\mathrm{Y}^{n} u\right)-\left(\mathrm{Y}^{n} v\right)\right\|_{\infty} \leq A_{n}\|u-v\|_{\infty}, \quad u, v \in C_{b}([0, \infty): X), n \in \mathbb{N},
$$


the well known extension of the Banach contraction principle yields that the mapping $Y(\cdot)$ has a unique fixed point. This completes the proof.

Let $\Omega$ be a bounded domain in $\mathbb{R}^{n}, b>0, m(x) \geq 0$ a.e. $x \in \Omega, m \in L^{\infty}(\Omega), 1<p<\infty$ and $X:=L^{p}(\Omega)$. Suppose that the operator $A:=\Delta-b$ acts on $X$ with the Dirichlet boundary conditions, and that $B$ is the multiplication operator by the function $m(x)$. Then, we can apply Theorem 4 with $\mathcal{A}=A B^{-1}$ in the study of existence and uniqueness of bounded quasi-asymptotically uniformly recurrent solutions of the semilinear fractional Poisson heat equation

$$
\begin{array}{r}
\mathbf{D}_{t}^{\gamma}[m(x) v(t, x)]=(\Delta-b) v(t, x)+f(t, m(x) v(t, x)), \quad t \geq 0, x \in \Omega ; \\
v(t, x)=0, \quad(t, x) \in[0, \infty) \times \partial \Omega, \\
m(x) v(0, x)=u_{0}(x), \quad x \in \Omega .
\end{array}
$$

It is also worth noting that we can apply Theorem 4 in the analysis of existence and uniqueness of bounded quasi-asymptotically uniformly recurrent solutions of the following fractional semilinear equation with higher order differential operators in the Hölder space $X=C^{\alpha}(\bar{\Omega})$ :

$$
\left\{\begin{array}{l}
\mathbf{D}_{t}^{\gamma} u(t, x)=-\sum_{|\beta| \leq 2 m} a_{\beta}(t, x) D^{\beta} u(t, x)-\sigma u(t, x)+f(t, u(t, x)), t \geq 0, x \in \Omega \\
u(0, x)=u_{0}(x), \quad x \in \Omega
\end{array}\right.
$$

where $\alpha \in(0,1), m \in \mathbb{N}, \Omega$ is a bounded domain in $\mathbb{R}^{n}$ with boundary of class $C^{4 m}, D^{\beta}=\prod_{i=1}^{n}\left(\frac{1}{i} \frac{\partial}{\partial x_{i}}\right)^{\beta_{i}}$, the functions $a_{\beta}: \bar{\Omega} \rightarrow \mathbb{C}$ satisfy certain conditions and $\sigma>0$ is sufficiently large. For more details, see Reference [5].

Basically, our results on the invariance of generalized quasi-asymptotical almost periodicity and uniform recurrence, established in Section 3.3, can be applied at any place where the variation of parameters formula takes effect. Furthermore, it is worth noting that the important examples presented in the monograph by Zaidman [7] (Examples 4, 5, 7, 8; pp. 32-34) have been used many times before, by numerous authors, to provide certain applications to the abstract PDEs. For our purposes, it will be very important to reexamine Reference [7] (Example 5). It is well known that the unique regular solution of the wave equation $u_{x x}(x, t)=u_{t t}(x, t), x \in \mathbb{R}, t \geq 0$, accompanied with the initial conditions $u(x, 0)=f(x), x \in \mathbb{R}, u_{t}(x, 0)=g(x), x \in \mathbb{R}$, is given by the famous d'Alembert formula

$$
u(x, t):=\frac{1}{2}[f(x+t)+f(x-t)]+\frac{1}{2} \int_{x-t}^{x+t} g(s) d s, \quad x \in \mathbb{R}, t \geq 0 .
$$

Let $t_{0}>0$ be a fixed real number. If the function $f(\cdot)$ is quasi-asymptotically uniformly recurrent, resp. $g(\cdot)$ is quasi-asymptotically uniformly recurrent, then the function $x \mapsto 1 / 2\left[f\left(x+t_{0}\right)+f\left(x-t_{0}\right)\right]$, $x \in \mathbb{R}$, resp.

$$
H_{t_{0}}(x):=\frac{1}{2} \int_{x-t_{0}}^{x+t_{0}} g(s) d s, \quad x \in \mathbb{R},
$$

is likewise quasi-asymptotically uniformly recurrent; this can be shown as in Reference [7]. Their sum will be quasi-asymptotically uniformly recurrent provided that these functions share the same sequence $\left(\alpha_{n}\right)$ in Definition 9.

\section{Conclusions}

In this paper, we analyzed several different classes of almost periodic type functions and uniformly recurrent type functions in Lebesgue spaces with variable exponents. The class of quasi-asymptotically uniformly recurrent functions was also introduced and systematically analyzed. We investigated the invariance of generalized almost periodicity and generalized uniform recurrence with variable 
exponents under the actions of convolution products, among many other themes, providing also several applications to the abstract (semilinear) Volterra integro-differential inclusions in Banach spaces.

Author Contributions: Writing-original draft, M.K. and W.-S.D. All authors have read and agreed to the published version of the manuscript.

Funding: The first author is partially supported by grant 174024 of Ministry of Science and Technological Development, Republic of Serbia. The second author is supported by Grant No. MOST 107-2115-M-017-004-MY2 of the Ministry of Science and Technology of the Republic of China.

Acknowledgments: The authors wish to express their hearty thanks to the anonymous referees for their valuable suggestions and comments.

Conflicts of Interest: The authors declare no conflict of interest.

\section{References}

1. Besicovitch, A.S. Almost Periodic Functions; Dover Publications: New York, NY, USA, 1954.

2. Diagana, T. Almost Automorphic Type and Almost Periodic Type Functions in Abstract Spaces; Springer: New York, NY, USA, 2013.

3. Fink, A.M. Almost Periodic Differential Equations; Springer: Berlin, Germany, 1974.

4. N'Guérékata, G.M. Almost Automorphic and Almost Periodic Functions in Abstract Spaces; Kluwer Academic Publishers: Dordrecht, The Netherlands, 2001.

5. Kostić, M. Almost Periodic and Almost Automorphic Type Solutions to Integro-Differential Equations; W. de Gruyter: Berlin, Germany, 2019.

6. Levitan, M. Almost Periodic Functions; G.I.T.T.L.: Moscow, Russia, 1959. (In Russian)

7. Zaidman, S. Almost-Periodic Functions in Abstract Spaces; Pitman Research Notes in Mathematics; Pitman: Boston, MA, USA, 1985; Volume 126.

8. Diagana, T.; Zitane, M. Weighted Stepanov-like pseudo-almost periodic functions in Lebesgue space with variable exponents $L^{p(x)}$. Afr. Diaspora J. Math. 2013, 15, 56-75.

9. Diagana, T.; Zitane, M. Stepanov-like pseudo-almost automorphic functions in Lebesgue spaces with variable exponents $L^{p(x)}$. Electron. J. Differ. Equ. 2013, 188, 20.

10. Diagana, T.; Kostić, M. Almost periodic and asymptotically almost periodic type functions in Lebesgue spaces with variable exponents $L^{p(x)}$. Filomat in press.

11. Diagana, T.; Kostić, M. Almost automorphic and asymptotically almost automorphic type functions in Lebesgue spaces with variable exponents $L^{p(x)}$. In Recent Studies in Differential Equations; Nova Science Publishers: New York, NY, USA, in press.

12. Kostić, M. Asymptotically Weyl almost periodic functions in Lebesgue spaces with variable exponents. arXiv 2020, arXiv:2001.08080.

13. Kostić, M.; Du, W.-S. Generalized almost periodicity in Lebesgue spaces with variable exponents. Mathematics 2020, 8, 928. [CrossRef]

14. Kostić, M. Quasi-asymptotically almost periodic functions and applications. Bull. Brazilan Math. Soc. 2020. Available online: https:/ /link.springer.com/article/10.1007/s00574-020-00197-7 (accessed on 31 January 2020). [CrossRef]

15. Kostić, M. Erratum and addendum to the paper "Weyl-almost periodic and asymptotically Weyl-almost periodic properties of solutions to linear and semilinear abstract Volterra integro-differential equations" Mat. Zam. SVFU, 25, N 2, 65-84 (2018). Math. Notes NEFU 2019, 26, 60-64.

16. Haraux. A.; Souplet, P. An example of uniformly recurrent function which is not almost periodic. J. Fourier Anal. Appl. 2004, 10, 217-220. [CrossRef]

17. Henríquez, H.R.; Pierri, M.; Táboas, P. On $S$-asymptotically $\omega$-periodic functions on Banach spaces and applications. J. Math. Appl. Anal. 2008, 343, 1119-1130. [CrossRef]

18. Diening, L.; Harjulehto, P.; Hästüso, P.; Ruzicka, M. Lebesgue and Sobolev Spaces with Variable Exponents; Lecture Notes in Mathematics; Springer: Heidelberg, Germany, 2011.

19. Fan, X.L.; Zhao, D. On the spaces $L^{p(x)}(O)$ and $W^{m, p(x)}(O)$. J. Math. Anal. Appl. 2001, 263, 424-446. [CrossRef]

20. Nguyen, P.Q.H. On Variable Lebesgue Spaces. Ph.D. Thesis, Kansas State University, Manhattan, KS, USA, 2011. 
21. Kostić, M. Almost Periodic Type Functions and Densities. Available online: https://hal.archives-ouvertes. $\mathrm{fr} /$ hal-02523952 (accessed on 29 March 2020).

22. Ruess, W.M.; Summers, W.H. Integration of Asymptotically almost Periodic Functions and Weak Asymptotic almost Periodicity; Instytut Matematyczny Polskiej Akademi Nauk: Warsaw, Poland, 1989; Volume 279, p. 35.

23. Fink, A.M. Extensions of almost automorphic sequences. J. Math. Anal. Appl. 1969, 27, 519-523. [CrossRef]

24. Kostić, M. Composition principles for almost periodic type functions and applications. J. Fract. Calc. Appl. submitted.

25. Favini, A.; Yagi, A. Degenerate Differential Equations in Banach Spaces; Chapman and Hall/CRC Pure and Applied Mathematics: New York, NY, USA, 1998.

26. Kostić, M. Abstract Degenerate Volterra Integro-Differential Equations; Mathematical Institute SANU: Belgrade, Serbia, 2020.

27. Kilbas, A.A.; Srivastava, H.M.; Trujillo, J.J. Theory and Applications of Fractional Differential Equations; Elsevier Science B.V.: Amsterdam, The Netherlands, 2006.

(C) 2020 by the authors. Licensee MDPI, Basel, Switzerland. This article is an open access article distributed under the terms and conditions of the Creative Commons Attribution (CC BY) license (http://creativecommons.org/licenses/by/4.0/). 